\title{
Scaling Behaviour in the Bose Gas
}

\author{
M. Broidioi ${ }^{\star}$, A. Verbeure ${ }^{\star \star}$ \\ K.U. Leuven, Instituut voor Theoretische Fysica, Celestijnenlaan 200 D, B-3001 Leuven, Belgium
}

Received: 24 October 1994

\begin{abstract}
The scaling behaviour of fluctuations of the Bose fields $\Phi(f)$ in the ergodic infinite volume equilibrium states of a $d$-dimensional Bose gas at temperature $T$ and density $\bar{\rho}$, can be classified in terms of the testfunctions $f$. In the low density regime, the space of testfunctions splits up in two subspaces, leading to two different types of non-commuting macroscopic field fluctuation observables. Testfunctions $f$ with Fourier transform $\hat{f}(0) \neq 0$ yield normal fluctuation observables. The local fluctuations of the field operators $\Phi(f)$ must be scaled subnormally (i.e. with a negative scaling index) if the testfunction $f$ has $\hat{f}(0)=0$. The macroscopic fluctuations of these fields can then again be described by a Bose field. The situation changes when the density of the gas exceeds the critical density. The field operators which have normal fluctuations in the low density regime need to be scaled abnormally in the high density regime, yielding classical macroscopic fluctuation observables. Another difference with the low density regime is that the space of testfunctions with $\hat{f}(0)=0$ splits up in two subspaces when the critical density is reached: for a first subspace the algebraic character of the macroscopic field fluctuation observables is also classical because it is necessary to scale the fluctuations of the field operators normally, while for the remaining subclass, the same negative scaling index is required as in the low density regime and hence also the algebraic character of these macroscopic fluctuations is again CCR.
\end{abstract}

\section{Introduction}

In the temperature-density $(T, \bar{\rho})$ phase diagram of a free Bose gas, one distinguishes a low density regime and a high density regime separated by a line of critical densities $\bar{\rho}=\rho_{c}(T, d)$. In the low density regime and on the critical line there is a unique gauge invariant ergodic infinite volume equilibrium state for each inverse temperature $\beta$ and density $\bar{\rho}$.

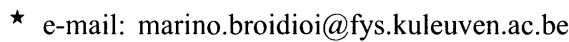

$\star \star$ e-mail: andre.verbeure@fys.kuleuven.ac.be 
However, if one steadily increases the density at a fixed inverse temperature $\beta$, then the gauge symmetry breaks down spontaneously once the density exceeds the critical density $\rho_{c}(\beta, d)$. The symmetry breaking generates a multiplicity of ergodic equilibrium states and for each one of them a macroscopic fraction of the particles condensates in the lowest energy level. This macroscopic collective phenomenon is called Bose condensation. Suitable order parameters for this phase transition are the space averages of the Boson field operators.

The model and its phase diagram will be presented in detail in the first section. The space averages of the field operators are the topic of the second section: they define suitable order parameters for the second order phase transition in a free Bose gas. The last and most important section deals with the classification of the fluctuations of the field operators $\Phi(f)$.

It is not simple to give a rigorous definition for the macroscopic observable characterising the fluctuations of a microscopic quantum observable; see e.g. [9, 10] where the case of normally scaled macroscopic fluctuations is discussed for a general quantum lattice system.

Definition 1. The local fluctuations with scaling index $\delta_{f}$ of a Bose field operator $\Phi(f)$ in an extremal infinite volume equilibrium state $\langle\cdot\rangle$ at arbitrary temperature $T$ and density $\bar{\rho}$ in a d-dimensional ball $B^{d}(0, R)$ of radius $R$ and centered in the origin, is defined by

$$
F_{R}^{\delta_{f}}(\Phi(f))=\frac{1}{\operatorname{vol}\left(B^{d}(0, R)\right)^{\left(\frac{1}{2}+\delta_{f}\right)}} \int_{B^{d}(0, R)} d x \tau_{x}\{\Phi(f)-\langle\Phi(f)\rangle\} .
$$

The $\tau_{x}$ denote the automorphisms of the microscopic CCR algebra associated with the space translations over vectors $x \in \mathbb{R}^{d}$.

The purpose of the scaling index $\delta_{f}$ is to obtain non-trivial limiting characteristic functions

$$
\lim _{R \rightarrow \infty}\left\langle\mathrm{e}^{\mathrm{i} s F_{R}^{\delta_{f}}(\Phi(f))}\right\rangle=\phi_{f}(s) \neq 1
$$

If this is the case, it is possible to identify in a canonical way the so-called limiting observables " $\lim _{R \rightarrow \infty} F_{R}^{\delta_{f}}(\Phi(f))$ " with well-defined operators $F^{\delta_{f}}(\Phi(f))$ acting on a Hilbert space. This way it makes sense to speak about the algebra of macroscopic field fluctuations.

If the $\delta_{f}=0$ and the $\phi_{f}(s)$ are Gaussian, one speaks about a system with normal fluctuations. If on the other hand it is necessary to use scaling indices $\delta_{f}>0$ or $\delta_{f}<0$, then the fluctuations are said to be respectively abnormal or subnormal.

Goderis et al. proved $[9,10,11]$ : if all self adjoint operators $A$ of a real linear space $A \in \mathscr{B} \subseteq \mathscr{A}_{L}$ (the algebra of strictly local observables), have normal fluctuations; i.e. the limits

$$
\lim _{R \rightarrow \infty} \omega\left(\mathrm{e}^{\mathrm{i} s F_{R}^{0}(A)}\right)=\exp \left[-s^{2} \sum_{x \in \mathbb{Z}^{d}}\left\{\omega\left(A \tau_{x} A\right)-\omega(A)^{2}\right\}\right]
$$

are defined, then one can identify the " $\lim _{R} F_{R}^{0}(A)$ " with Bose field operators $F^{0}(A)$ of a macroscopic CCR algebra with a macroscopic quasi-free state $\tilde{\omega}$ induced by 
the $\lim _{R} \omega\left(\mathrm{e}^{\mathrm{i} s F_{R}^{0}(A)}\right)$. The Bose fields obey the commutation relations:

$$
\left[F^{0}(A), F^{0}(B)\right]=i \sigma(A, B)=\lim _{R \rightarrow \infty} \omega\left(\left[F_{R}^{0}(A), F_{R}^{0}(B)\right]\right)
$$

Under rather weak conditions on the space clustering of the state $\omega$ (see [10]), the total algebra of strictly local observables $\mathscr{A}_{L}$ has normal fluctuations. For further details we refer to the literature $[9,10,11]$.

Concerning the algebraic structure of abnormal and subnormal fluctuations, there exist partial results for the anharmonic crystal [15]. In this paper it is explained how the commutator of two local fluctuations $F_{R}^{\delta_{A}}(A)$ and $F_{R}^{\delta_{B}}(B)$ goes to zero if $\delta_{A}+\delta_{B}>0$ or to the $c$-number $\omega\left(\left[A, \sum_{x \in \mathbb{Z}^{d}} \tau_{x} B\right]\right)$ if $\delta_{A}+\delta_{B}=0$. For the observables studied in [15], it was irrelevant to study the algebraic character of the commutator of two fluctuations $F^{\delta_{A}}(A)$ and $F^{\delta_{B}}(B)$ with $\delta_{A}+\delta_{B}<0$. However, the results on subnormally scaled fluctuations of field operators already indicate that it should be possible to define non-trivial commutation relations of macroscopic fluctuations in situations where $\delta_{A}+\delta_{B}<0$. This topic is discussed in general in [7].

Finally we mention that the scaling indices $\delta_{f}$ obtained in this paper differ from those obtained in [5] where the infinite volume limits are taken of local fluctuations of the field operators in the local equilibrium states. The indices $\delta_{f}$ obtained in the present setup correspond to the familiar critical exponents $\eta$ of the susceptibility, which is known to be zero for free and for mean field models.

We present here rigorous results on the phenomenon of finite size scaling.

\section{The Model}

\subsection{The algebra of observables for bosons.}

The first successful treatment of an infinitely extended Bose gas using the theory of states on CCR algebras dates back to 1963 [2]. Using the method of second quantisation [4], we derive a representation of the CCR algebra appropriate to describe a $d$-dimensional Bose gas.

The Bose-Fock space $\mathscr{F}$ on $\mathbb{R}^{d}$ is the completed direct sum $\mathscr{F}=\bigoplus_{n \geqq 0} \mathscr{F}^{(n)}$, where $\mathscr{F}^{(0)}=\mathbb{C}$ and $\mathscr{F}^{(n)}=L_{\text {symm }}^{2}\left(\mathbb{R}^{(d n)}\right)$ and consider the subspace $\mathscr{G}$ which is the incomplete direct sum:

$$
\mathscr{G}=\bigcup_{l=1}^{\infty} \bigoplus_{n=0}^{l}\left(\mathscr{F}^{(n)} \bigcap C_{\text {comp }}^{\infty}\left(\mathbb{R}^{d n}\right)\right) \subset \mathscr{F} .
$$

The creation and annihilation operators on $\mathscr{G}$ are: for $f \in \mathscr{F}^{(1)}$,

$$
\begin{aligned}
\left(a^{+}(f) \psi\right)^{(n)}\left(x_{1}, \ldots, x_{n}\right) & =\frac{1}{\sqrt{n}} \sum_{j=1}^{n} f\left(x_{j}\right) \psi^{(n-1)}\left(x_{1}, \ldots, \widehat{x}_{j}, \ldots, x_{n}\right), \\
(a(f) \psi)^{(n)}\left(x_{1}, \ldots, x_{n}\right) & =\sqrt{n+1} \int d x_{1} \overline{f\left(x_{1}\right)} \psi^{(n+1)}\left(x_{1}, \ldots, x_{n}\right)
\end{aligned}
$$

with $\psi^{(n)}=P^{n} \psi$, where $P^{n}$ is the orthogonal projection of $\mathscr{F}$ onto $\mathscr{F}^{(n)}$. The following commutation relations are easily verified for these operators on $\mathscr{G}$ :

$$
\left[a(f), a^{+}(g)\right]=(f, g) \mathbb{1} .
$$


The Bose field operators, defined as the sum of creation and annihilation operators,

$$
\Phi(f)=\frac{1}{\sqrt{2}}\left\{a^{+}(f)+a(f)\right\}
$$

are essentially self-adjoint [4] and hence when exponentiated, they yield the unitary operators

$$
W(f)=\exp [\mathrm{i} \Phi(f)]
$$

which satisfy the Weyl relations:

$$
W\left(f_{1}\right) W\left(f_{2}\right)=W\left(f_{1}+f_{2}\right) \exp \left[-\frac{\mathrm{i}}{2} \operatorname{Im}\left(f_{1}, f_{2}\right)\right] \text {. }
$$

The norm closure of the linear span of these operators $W(f), f \in C_{\text {comp }}^{\infty}\left(\mathbb{R}^{d}\right)$, forms a $C^{*}$-algebra $\mathfrak{U}$ containing the quasi-localised observables of a Boson gas.

\subsection{Ergodic equilibrium states of the free Bose gas.}

Araki and Woods explained how the infinitely extended Bose gases can be described in the framework of $C^{*}$-algebras [2]. In the same context they proposed a simple expression for the equilibrium state of the infinite free Bose gas, which later turned out to be the infinite volume limit of the canonical states. Lewis and Pule reformulated some ideas of $\mathrm{Kac}$ in this new framework and this lead to the infinite volume limit of the grand canonical states [13] which are linear combinations of canonical states at different densities. Kac had shown that the coefficients of this linear combination converge to a simple distribution (in the infinite volume limit), the so-called Kac density. Lewis and Pule realised that the infinite volume limit state they had calculated from local grand canonical states, was related to the one conjectured by Araki and Woods through this Kac density, but the proof of convergence of the canonical states is due to Cannon [8]. A first important result on critical behaviour in Bose systems is about the density fluctuations in the canonical states in [3]. No attention is paid to the algebraic character of the fluctuation observables.

For free Bose gases with particle densities above sharply determined critical values, the limiting canonical and grand canonical states are not equivalent: the Kac density is not a delta function. Hence it is useful to determine the ergodic equilibrium states. This will be done in this section using the well known technique of adding an external field term to the local Hamiltonians.

Let $\Lambda^{1}$ be an arbitrary bounded region in $\mathbb{R}^{d}(d \geqq 3)$ of unit volume. $\Lambda^{1}$ must be star shaped with respect to some interior point, chosen to be the origin. The boundary $\partial \Lambda^{1}$ has to satisfy certain regularity conditions [14].

Isotropic dilation of this unit volume defines the sequence of regions $\Lambda^{L}$ for $L>0$, by:

$$
\Lambda^{L}=\left\{x \in \mathbb{R}^{d}: L^{-1} x \in \Lambda^{1}\right\}
$$

and put $\mathscr{H}^{L}=L^{2}\left(\Lambda^{L}\right)$. One has $\Lambda^{L} \subset \Lambda^{L^{\prime}}$ and $\mathscr{H}^{L} \subset \mathscr{H}^{L^{\prime}}$ whenever $L<L^{\prime}$.

Take $H^{L}$ to be the unique self adjoint operator on $\mathscr{H}^{L}$ determined by the Laplacian $-\frac{\Delta}{2}$ in $\Lambda^{L}$ and by Dirichlet boundary conditions $\phi=0$ on $\partial \Lambda^{L}$ and let $\left\{\phi_{k}^{L}\right\}_{k=1,2, \ldots}$ be an orthonormal set of eigenfunctions labeled by the index $k$ such that the corresponding eigenvalues $0 \leqq E_{1}^{L}<E_{2}^{L} \leqq E_{3}^{L} \leqq \ldots$. The set $\left\{\phi_{k}^{L} \mid k=1,2, \ldots\right\}$ forms a complete orthonormal set of $\mathscr{H}^{L}$. 
The local Hamiltonians describing a gas of free bosons in a volume $\Lambda^{L}$ with particle density $\bar{\rho}$ are induced by these $H^{L}$. They are specified by the following operators acting on the Fock space $\mathscr{F}\left(\mathscr{H}^{L}\right)$ :

$$
\begin{aligned}
\tilde{H}_{\mu^{L}, h, 0}^{L}= & \sum_{k=1}^{\infty} E_{k}^{L} a^{+}\left(\phi_{k}^{L}\right) a\left(\phi_{k}^{L}\right)-\mu^{L} \sum_{k=1}^{\infty} a^{+}\left(\phi_{k}^{L}\right) a\left(\phi_{k}^{L}\right) \\
& +h L^{\frac{d}{2}}\left(\mathrm{e}^{-10} a^{+}\left(\phi_{1}^{L}\right)+\mathrm{e}^{\mathrm{i} \theta} a\left(\phi_{1}^{L}\right)\right) .
\end{aligned}
$$

The values for the chemical potentials $\mu^{L} \leqq E_{1}^{L}$ are determined by the constraint that the local equilibrium state $\omega_{\beta, \bar{\rho}, h, 0}^{L}(\cdot)=\frac{\mathbf{T r}\left(\exp \left[-\beta \tilde{H}_{\mu^{L}, h, 0}^{L}\right] \cdot\right)}{\operatorname{Tr}\left(\exp \left[-\beta \tilde{H}_{\mu}^{L} L_{, h, \theta}^{L}\right]\right)}$ at inverse temperature $\beta$ has particle density $\bar{\rho}$; i.e.

$$
\forall L: \omega_{\beta, \bar{\rho}, h, \theta}^{L}\left(L^{-d} \sum_{k=1}^{\infty} a^{+}\left(\phi_{k}^{L}\right) a\left(\phi_{k}^{L}\right)\right)=\bar{\rho}
$$

A gauge-symmetry-breaking field $h>0, \theta \in[0,2 \pi)$ is included in the Hamiltonians to recover the extremal translation invariant infinite volume equilibrium states.

The generating functionals of the local equilibrium states at inverse temperature $\beta$ and density $\bar{\rho}$ are given by the Gibbs states:

$$
E_{\beta, \bar{\rho}, h, \theta}^{L}(f) \equiv \omega_{\beta, \bar{\rho}, h, \theta}^{L}(W(f)) .
$$

By the orthogonality of the $\phi_{k}^{L}$, the algebra $\mathfrak{U}$ is equivalent to the tensor product $\bigotimes_{k=1}^{\infty} \mathfrak{U}_{k}$ and in this representation

where

$$
\exp \left[-\beta \tilde{H}_{\mu^{L}, h, 0}^{L}\right]=\bigotimes_{k=1}^{\infty} \exp \left[-\beta \tilde{H}_{\mu^{L}, h, 0}^{L}(k)\right]
$$

$$
\tilde{H}_{\mu^{L}, h, \theta}^{L}(k)=\left(E_{k}^{L}-\mu^{L}\right) a^{+}\left(\phi_{k}^{L}\right) a\left(\phi_{k}^{L}\right)+\delta_{k, 1} h L^{\frac{d}{2}}\left(\mathrm{e}^{-\mathrm{i} \theta} a^{+}\left(\phi_{1}^{L}\right)+\mathrm{e}^{\mathrm{i} \theta} a\left(\phi_{1}^{L}\right)\right) .
$$

Furthermore

Hence

$$
W(f)=\bigotimes_{k=1}^{\infty} \exp \left[\frac{\mathrm{i}}{\sqrt{2}}\left\{\left(\phi_{k}^{L}, f\right) a^{+}\left(\phi_{k}^{L}\right)+\overline{\left(\phi_{k}^{L}, f\right)} a\left(\phi_{k}^{L}\right)\right\}\right] .
$$

$$
\begin{aligned}
& E_{\beta, \bar{\rho}, h, \theta}^{L}(f)=\exp {\left[\frac{-\mathrm{i} \beta h L^{\frac{d}{2}}}{\sqrt{2} \ln \zeta_{h}^{L}}\left\{\mathrm{e}^{\mathrm{i} \theta}\left(\phi_{1}^{L}, f\right)+\mathrm{e}^{-1 \theta} \overline{\left(\phi_{1}^{L}, f\right)}\right\}\right] } \\
& \times \exp \left[-\frac{1}{4}\|f\|^{2}\right] \exp \left[-\frac{1}{2} \mathscr{A}_{\beta, \bar{\rho}, h, \theta}^{L}(f, f)\right] .
\end{aligned}
$$

The form

$$
\mathscr{A}_{\beta, \bar{\rho}, h, 0}^{L}(f, f)=\left(f, \frac{\zeta_{h}^{L} \mathbb{1}}{\exp \left[\beta\left(H^{L}-E_{1}^{L} \mathbb{1}\right)\right]-\zeta_{h}^{L} \mathbb{1}} f\right),
$$

where

$$
\zeta_{h}^{L} \equiv \exp \left[\beta\left(\mu_{h}^{L}-E_{1}^{L}\right)\right] \in[0,1]
$$


is determined by the density and is the unique solution of:

$$
\bar{\rho}=\frac{\beta^{2} h^{2}}{(\ln \zeta)^{2}}+L^{-d} \sum_{k=1}^{\infty} \frac{\zeta}{\mathrm{e}^{\beta \eta_{k}^{L}}-\zeta},
$$

where we used the notation $\eta_{k}^{L}=E_{k}^{L}-E_{1}^{L}$.

The first step in finding extremal infinite volume equilibrium states, consists in taking the limit $L \rightarrow \infty$ of the generating functionals $E_{\beta, \bar{\rho}, h, \theta}^{L}$ for fixed parameters $\beta, \bar{\rho}, h, \theta$. Let $g_{\alpha}$ be the function defined on the interval $[0,1]$ by:

$$
g_{\alpha}(z)=\sum_{n=1}^{\infty} \frac{z^{n}}{n^{\alpha}} \quad \alpha>1
$$

and let $\rho_{c}(\beta, d)$ be the "critical densities" defined by:

$$
\rho_{c}(\beta, d)=(2 \pi \beta)^{-\frac{d}{2}} g_{\frac{d}{2}}(1) .
$$

Theorem 1. The generating functional $E_{\beta, \bar{\rho}, h, \theta}^{\infty}=\lim _{L \rightarrow \infty} E_{\beta, \bar{\rho}, h, \theta}^{L}$ is given for $f \in$ $\mathscr{D}=C_{\text {comp }}^{\infty}\left(\mathbb{R}^{d}\right)$ by:

$$
\begin{aligned}
E_{\beta, \bar{\rho}, h, \theta}^{\infty}(f)= & \exp \left[\frac{-\mathrm{i} \beta h(2 \pi)^{\frac{d}{2}}}{\sqrt{2} \ln \zeta_{h}^{\infty}}\left\{\mathrm{e}^{\mathrm{i} \theta} \overline{\phi_{1}^{1}(0)} \hat{f}(0)+\mathrm{e}^{-\mathrm{i} \theta} \phi_{1}^{1}(0) \overline{\hat{f}(0)}\right\}\right] \\
& \times \exp \left[-\frac{1}{4}\|f\|^{2}\right] \times \exp \left[-\frac{1}{2} \mathscr{A}_{\beta, \bar{\rho}, h, \theta}^{\infty}(f, f)\right]
\end{aligned}
$$

with

$$
\begin{aligned}
\mathscr{A}_{\beta, \bar{\rho}, h, 0}^{\infty} & =\int d x \overline{f(x)} \int d y\left[(2 \pi \beta)^{-\frac{d}{2}} \sum_{n=1}^{\infty} \frac{\mathrm{e}^{-\frac{\|x-y\|^{2}}{2 n \beta}}}{n^{\frac{d}{2}}}\left(\zeta_{h}^{\infty}\right)^{n}\right] f(y) \\
& =(2 \pi)^{-d} \int d p|\hat{f}(p)|^{2} \frac{\zeta_{h}^{\infty}}{\mathrm{e}^{\beta|p|^{2}-\zeta_{h}^{\infty}}}
\end{aligned}
$$

and $\zeta_{h}^{\infty}$ the unique solution of the equation:

$$
\begin{aligned}
\bar{\rho} & =\frac{\beta^{2} h^{2}}{\left(\ln \zeta_{h}^{\infty}\right)^{2}}+(2 \pi \beta)^{-\frac{d}{2}} g_{\frac{d}{2}}(\zeta), \\
\hat{f}(p) & =(2 \pi)^{-\frac{d}{2}} \int_{\mathbb{R}^{d}} d x f(x) \mathrm{e}^{\mathrm{i} p x} .
\end{aligned}
$$

The second step is to take the limit $h \rightarrow 0+$ of the $E_{\beta, \bar{\rho}, h, \theta}^{\infty}$, keeping the other parameters $\beta, \bar{\rho}, \theta$ fixed.

Theorem 2. The generating functional $E_{\beta, \bar{\rho}, \theta}^{\infty}=\lim _{h \rightarrow 0+} E_{\beta, \bar{\rho}, h, \theta}^{\infty}$ is given on $\mathscr{D}$ by:

1. $\bar{\rho}<\rho_{c}(\beta)$

$$
E_{\beta, \bar{\rho}}^{\infty}(f)=\exp \left[\frac{-1}{4}\|f\|^{2}\right] \times \exp \left[\frac{-1}{2(2 \pi)^{d}} \int d p|\hat{f}(p)|^{2} \frac{\zeta}{\mathrm{e}^{\beta|p|^{2}}-\zeta}\right],
$$


where $\zeta$ is the unique solution of

$$
\bar{\rho}=(2 \pi \beta)^{-\frac{d}{2}} g_{\frac{d}{2}}(\zeta)
$$

The dependence on $\theta$ disappears in the limit and $E_{\beta, \bar{\rho}, \theta}^{\infty}$ is the generating functional of a gauge-invariant state.

\section{2. $\bar{\rho} \geqq \rho_{c}(\beta)$}

$$
\begin{aligned}
E_{\beta, \bar{\rho}, 0}^{\infty}(f)= & \exp \left[\frac{-\mathrm{i}(2 \pi)^{\frac{d}{2}}}{\sqrt{2}} \sqrt{\bar{\rho}-\rho_{c}(\beta)}\left\{\mathrm{e}^{\mathrm{i} \theta} \overline{\phi_{1}^{1}(0)} \hat{f}(0)+\mathrm{e}^{-10} \phi_{1}^{1}(0) \overline{\hat{f}(0)}\right\}\right] \\
& \times \exp \left[\frac{-1}{4}\|f\|^{2}\right] \times \exp \left[\frac{-1}{2(2 \pi)^{d}} \int d p|\hat{f}(p)|^{2} \frac{1}{\mathrm{e}^{\beta|p|^{2}}-1}\right] .
\end{aligned}
$$

The gauge-symmetry is broken for the corresponding states and there is clearly a dependency on the boundary conditions.

The proofs of both theorems are merely technical generalisations to arbitrary dimensions of the proofs presented in the paper on the grand canonical 3 dimensional Bose gas by Lewis and Pulè [13]. The presence of the external field is not a problem; for the proofs, see [6].

\section{The Order Parameters}

The spontaneous breaking of the gauge symmetry and the appearance of a Bose condensate is described with order parameters which are the infinite volume limits of the following operators: for any function $f \in \mathscr{D}=C_{\text {comp }}^{\infty}\left(\mathbb{R}^{d}\right)$, one can define in the GNS representations of the states $\omega_{\beta, \bar{\rho}, \theta}^{\infty}$ and $\omega_{\beta, \bar{\rho}}^{\infty}$, the operators

$$
\mathcal{O}_{\Lambda}(f)=\frac{1}{|\Lambda|} \int_{\Lambda} d x \tau_{x} \Phi(f)
$$

These operators $\mathcal{O}_{\Lambda}(f)$ are unbounded operators and therefore we prove explicitly that their limiting distributions in an ergodic equilibrium state agree with the predictions of the general theory on the weak law of large numbers [12], [4]:

Proposition 1. For volumes $\Lambda$, consisting of d-dimensional balls $B^{d}(0, R)$, centered in the origin and with increasing radius $R$ and volume $|\Lambda|=\operatorname{vol}\left(B^{d}(0, R)\right)$, the distributions of the order parameters in an extremal equilibrium state are:

1. if $\bar{\rho} \leqq \rho_{c}(\beta, d)$, then

$$
\lim _{|\Lambda| \rightarrow \infty} \omega_{\beta, \bar{\rho}}^{\infty}\left(\exp \left[i \mu \mathcal{O}_{\Lambda}(f)\right]\right)=1
$$

2. if $\bar{\rho} \geqq \rho_{c}(\beta, d)$, then

$$
\lim _{|\Lambda| \rightarrow \infty} \omega_{\beta, \bar{\rho}, \theta}^{\infty}\left(\exp \left[\mathrm{i} \mu \mathcal{O}_{\Lambda}(f)\right]\right)=\exp \left[\frac{-\mathrm{i} \mu}{\sqrt{2}} \sqrt{\bar{\rho}-\rho_{c}(\beta)}\left\{\mathrm{e}^{\mathrm{i} \theta} \overline{\phi_{1}^{1}(0)} \hat{f}(0)+c . c .\right\}\right] .
$$


Proof. Proving the statement for the high density regime $\bar{\rho} \geqq \rho_{c}(\beta, d)$ immediately implies the result for the low density regime. Thus consider any state $\omega_{\beta, \bar{\rho}, 0}^{\infty}$ with $\bar{\rho} \geqq \rho_{c}(\beta, d)$ and $\theta \in[0,2 \pi)$. From Theorem 2 , one has:

$$
\begin{aligned}
& \lim _{|\Lambda| \rightarrow \infty} \omega_{\beta, \bar{\rho}, \theta}^{\infty}\left(\exp \left[\mathrm{i} \mu \mathcal{O}_{\Lambda}(f)\right]\right) \\
&=\lim _{R \rightarrow \infty} \omega_{\beta, \bar{\rho}, \theta}^{\infty}\left(\exp \left[\frac{\mathrm{i} \mu}{\operatorname{vol}\left(B^{d}(0, R)\right)_{B^{d}(0, R)}} d x \tau_{x} \Phi(f)\right]\right) \\
&=\lim _{R \rightarrow \infty} \omega_{\beta, \bar{\rho}, \theta}^{\infty}\left(\exp \left[\mathrm{i} \Phi\left(\frac{\mu}{\operatorname{vol}\left(B^{d}(0, R)\right)_{B^{d}(0, R)}} \tau_{x} f\right)\right]\right) \\
&=\lim _{R \rightarrow \infty} \exp \left[\frac{-\mathrm{i} \mu(2 \pi)^{\frac{d}{2}}}{\sqrt{2}} \sqrt{\bar{\rho}-\rho_{c}(\beta)}\left\{\mathrm{e}^{\mathrm{i} \theta} \frac{\phi_{1}^{1}(0)}{g_{R}}(0)+\text { c.c. }\right\}\right] \\
& \times \lim _{R \rightarrow \infty} \exp \left[-\frac{\mu^{2}}{4}\left\|g_{R}\right\|^{2}\right] \\
& \times \lim _{R \rightarrow \infty} \exp \left[-\frac{\mu^{2}}{2}(2 \pi)^{-d} \int d p\left|\hat{g}_{R}(p)\right|^{2} \frac{1}{\mathrm{e}^{\beta|p|^{2}}-1}\right],
\end{aligned}
$$

where

$$
\left(\tau_{x} f\right)(y)=f^{x}(y)=f(y-x),
$$

and where $g_{R}$ is a shorthand notation for the function

$$
g_{R}(y)=\frac{1}{R^{d} V_{d^{d}}} \int_{B^{d}(0, R)} d x f(y-x),
$$

with

$$
V_{d}=\operatorname{vol}\left(B^{d}(0,1)\right)
$$

An easy calculation:

$$
\begin{aligned}
\hat{g}_{R}(0) & =\frac{(2 \pi)^{-\frac{d}{2}}}{R^{d} V_{d}} \int d y \int_{B^{d}(0, R)} d x f(y-x) \\
& =\frac{1}{R^{d} V_{d}} \int_{B^{d}(0, R)} d x(2 \pi)^{-\frac{d}{2}} \int d y f(y) \\
& =\hat{f}(0)
\end{aligned}
$$

yields the limiting form of (3.6):

$$
\begin{aligned}
& \lim _{R \rightarrow \infty} \exp \left[\frac{-\mathrm{i} \mu(2 \pi)^{\frac{d}{2}}}{\sqrt{2}} \sqrt{\bar{\rho}-\rho_{c}(\beta)}\left\{\mathrm{e}^{\mathrm{i} \theta \overline{\phi_{1}^{1}(0)}} \hat{g}_{R}(0)+\mathrm{e}^{-\mathrm{i} \theta} \phi_{1}^{1}(0) \overline{\hat{g}_{R}(0)}\right\}\right] \\
& \quad=\exp \left[\frac{-\mathrm{i} \mu(2 \pi)^{\frac{d}{2}}}{\sqrt{2}} \sqrt{\bar{\rho}-\rho_{c}(\beta)}\left\{\mathrm{e}^{\mathrm{i} \theta} \overline{\phi_{1}^{1}(0)} \hat{f}(0)+\mathrm{e}^{-\mathrm{i} \theta} \phi_{1}^{1}(0) \overline{\hat{f}(0)}\right\}\right] .
\end{aligned}
$$


To handle the remaining factors (3.7) and (3.8), it is useful to calculate first the Fourier transform of $g_{R}$ :

$$
\begin{aligned}
\hat{g}_{R}(p) & =\frac{(2 \pi)^{-\frac{d}{2}}}{R^{d} V_{d}} \int d y \mathrm{e}^{\mathrm{i} p y} \int_{B^{d}(0, R)} d x f(y-x) \\
& =\frac{(2 \pi)^{-\frac{d}{2}}}{R^{d} V_{d}} \int_{B^{d}(0, R)} d x \mathrm{e}^{\mathrm{i} p x} \int d y f(y) \mathrm{e}^{\mathrm{i} p y} \\
& =\frac{(2 \pi)^{\frac{d}{2}}}{R^{d} V_{d}} \hat{\chi}_{B^{d}(0, R)}(p) \hat{f}(p),
\end{aligned}
$$

where

$$
\begin{aligned}
\hat{\chi}_{B^{d}(0, R)}(p) & =(2 \pi)^{-\frac{d}{2}} \int_{B^{d}(0, R)} d x \mathrm{e}^{\mathrm{i} p x} \\
& =(2 \pi)^{-\frac{d}{2}} \int d x \chi_{B^{d}(0, R)}(x) \mathrm{e}^{1 p x}
\end{aligned}
$$

It is straightforward to calculate $\hat{\chi}_{B^{d}(0, R)}(p)$ in spherical coordinates where the polar axis is in the direction of $p$ :

$$
\begin{aligned}
\hat{\chi}_{B^{d}(0, R)}(p) & =(2 \pi)^{-\frac{d}{2}} \Phi_{d-2} \int_{0}^{R} d r r^{d-1}\left(\frac{|p| r}{2}\right)^{-\frac{d-2}{2}} \Gamma\left(\frac{d-1}{2}\right) \sqrt{\pi} J_{\frac{d-2}{2}}(|p| r) \\
& =\frac{R^{\frac{d}{2}}}{|p|^{\frac{d}{2}}} J_{\frac{d}{2}}(|p| R),
\end{aligned}
$$

where $\Phi_{d-2}=\frac{2 \pi^{\frac{d-1}{2}}}{\Gamma\left(\frac{d-1}{2}\right)}$ is the area of a $(d-2)$ dimensional sphere. Formula (11.1.1) of [1] has been used in the last line.

The remaining factors (3.7) and (3.8) can now also be discussed.

Beginning with (3.7), one obtains:

$$
\lim _{R \rightarrow \infty}\left\|g_{R}\right\|^{2}=\frac{(2 \pi)^{d}}{V_{d}^{2}} \lim _{R \rightarrow \infty} R^{-d} \int d p\left|\hat{f}\left(\frac{p}{R}\right)\right|^{2} \frac{\left|J_{\frac{d}{2}}(|p|)\right|^{2}}{(|p|)^{d}}=0
$$

This limit is zero for $f \in \mathscr{D}$, by dominated convergence, since

$$
\int_{0}^{\infty} d|p||p|^{-1}\left|J_{\frac{d}{2}}(|p|)\right|^{2}=\frac{2}{d}
$$

(see formula (11.4.6) of [1]). 
The last factor (3.8) has a divergent factor in the integrand: $\frac{1}{\mathrm{e}^{\beta|p|^{2}-1}}$ is asymptotically $O\left(|p|^{-2}\right)$ around $p=0$.

$$
\begin{aligned}
& \lim _{R \rightarrow \infty} \int d p\left|\hat{g}_{R}(p)\right|^{2} \frac{1}{\mathrm{e}^{\beta|p|^{2}}-1} \\
& \quad=\frac{(2 \pi)^{d}}{V_{d}^{2}} \lim _{R \rightarrow \infty} R^{-d} \int d p \frac{\left|J_{\frac{d}{2}}(|p|)\right|^{2}}{|p|^{d}} \frac{\left|\hat{f}\left(\frac{p}{R}\right)\right|^{2}}{\mathrm{e}^{\beta \frac{|p|^{2}}{R^{2}}}-1} \\
& \quad=\lim _{\varepsilon \rightarrow 0+R \rightarrow \infty} \lim _{R \rightarrow \infty} \frac{(2 \pi)^{d}}{R^{d} V_{d}^{2}}\left[\int_{0 \leqq|p| \leqq \varepsilon R} d p+\int_{|p| \geqq \varepsilon R} d p\right] \frac{\left|J_{\frac{d}{2}}(|p|)\right|^{2}}{|p|^{d}} \frac{\left|\hat{f}\left(\frac{p}{R}\right)\right|^{2}}{\mathrm{e}^{\beta \frac{|p|^{2}}{R^{2}}}-1} .
\end{aligned}
$$

1. The contribution of the integration domain where $|p| \geqq \varepsilon R$, can be bounded

$$
\begin{aligned}
& R^{-d} \int_{|p| \geqq \varepsilon R} d p \frac{\left|J_{\frac{d}{2}}(|p|)\right|^{2}}{|p|^{d}} \frac{\left|\hat{f}\left(\frac{p}{R}\right)\right|^{2}}{\mathrm{e}^{\beta \frac{|p|^{2}}{R^{2}}}-1} \\
& \leqq R^{-d} M_{f} \frac{1}{\mathrm{e}^{\beta \varepsilon^{2}}-1} \int_{|p| \geqq \varepsilon R} \frac{\left|J_{\frac{d}{2}}(|p|)\right|^{2}}{(|p|)^{d}}
\end{aligned}
$$

and then of course also $\stackrel{R \rightarrow \infty}{\longrightarrow} 0$

$$
\lim _{\varepsilon \rightarrow 0+R \rightarrow \infty} \lim _{R \rightarrow \infty} R_{|p| \geqq \varepsilon R} d p \frac{\left|J_{\frac{d}{2}}(|p|)\right|^{2}}{|p|^{d}} \frac{\left|\hat{f}\left(\frac{p}{R}\right)\right|^{2}}{\mathrm{e}^{\beta \frac{|p|^{2}}{R^{2}}}-1}=0 .
$$

2. Finally, one also has that

This follows from a bound on

$$
\lim _{\varepsilon \rightarrow 0+R \rightarrow \infty} \lim _{0 \leqq|p| \leqq \varepsilon R} d p \frac{\left|J_{\frac{d}{2}}(|p|)\right|^{2}}{|p|^{d}} \frac{\left|\hat{f}\left(\frac{p}{R}\right)\right|^{2}}{\mathrm{e}^{\beta \frac{|p|^{2}}{R^{2}}}-1}=0 .
$$

$$
\begin{aligned}
& R_{0 \leqq|p| \leqq \varepsilon R}^{-d} \int_{0 \leqq|p| \leqq \varepsilon R} d p \frac{\left|J_{\frac{d}{2}}(|p|)\right|^{2}}{|p|^{d}} \frac{\left|\hat{f}\left(\frac{p}{R}\right)\right|^{2}}{\mathrm{e}^{\beta \frac{|p|^{2}}{R^{2}}}-1} \\
& \quad=R^{-d} \int_{|p|^{d}} d p \frac{\left|J_{\frac{d}{2}}(|p|)\right|^{2}}{\left|\hat{f}\left(\frac{p}{R}\right)\right|^{2}} \\
& \left.\quad \leqq R^{-d+2} \Phi_{d-1} M_{f} \beta^{-1} \int_{0}^{\varepsilon R} d|p| \frac{\mathrm{e}^{\beta \frac{|p|^{2}}{R^{2}}}-1}{\beta \frac{|p|^{2}}{R^{2}}}\right) \\
& \stackrel{\left|J_{\frac{d}{2}}(|p|)\right|^{2}}{|p|^{3}} \\
& \stackrel{R \rightarrow \infty}{\longrightarrow} 0
\end{aligned}
$$

because $d \geqq 3$.

This completes the proof of Proposition (1). 


\section{Zero Mode Fluctuations of Field Operators}

At the critical densities, the increased importance of long-range correlations manifests itself through the formation of a Bose condensate which is a phenomenon of macroscopic order. Another effect of the long-range correlations is that a deviation from equilibrium in a small region of the system, will have an influence at very large distances. It is logical to expect that these long-range correlations lead to abnormal behaviour for the fluctuations of the field operators in the $k=0$ mode; i.e. for

$$
F_{R}^{\delta_{f}}(\Phi(f))=\left(R^{d} V_{d}\right)^{-\left(\frac{1}{2}+\dot{\delta}_{f}\right)} \int_{B^{d}(0, R)} d x\left\{\tau_{x} \Phi(f)-\omega_{\beta, \bar{\rho}, \theta}^{\infty}\left(\tau_{x} \Phi(f)\right)\right\}
$$

when the density $\bar{\rho}$ of the state exceeds the critical density.

In this section, central limit theorems and reconstruction theorems will be formulated and proved. In every proposition and theorem on the field fluctuations, it is implicitly assumed that the testfunctions $f$ are Schwartz functions with Fourier transforms $\hat{f}(p)$ which are analytic in a neighbourhood of $p=0$.

\subsection{The low density regime}

Low density regimes have usually exponential clustering properties, hence it is not surprising that the fluctuations are normal for most operators [10].

Proposition 2. For densities $\bar{\rho}<\rho_{c}(\beta, d)$, the $k=0$ mode fluctuations of field operators $\Phi(f)$ are normal for testfunctions $f$ with $\hat{f}(0) \neq 0$; i.e. $\delta_{f}=0$ and

$$
\lim _{R \rightarrow \infty} \omega_{\beta, \bar{\rho}}^{\infty}\left(\mathrm{e}^{\mathrm{i} s F_{R}^{0}(\Phi(f))}\right)=\exp \left[-s^{2}|\hat{f}(0)|^{2}\left[(2 \pi)^{d}+\frac{2 \zeta}{1-\zeta}\right]\right] .
$$

Proof. We have:

$$
\begin{aligned}
\omega_{\beta, \bar{\rho}}^{\infty}\left(\mathrm{e}^{\mathrm{i} s F_{R}^{0}(\Phi(f))}\right)= & \omega_{\beta, \bar{\rho}}^{\infty}\left(\mathrm{e}^{\mathrm{i} \Phi\left(\sqrt{2} s R^{-\frac{d}{2}} V_{d}^{-\frac{1}{2}} \int_{B^{d}(0, R)} d x f^{-x}\right)}\right) \\
= & \exp \left[-\frac{s^{2}}{2} R^{d} V_{d}\left\|g_{R}\right\|^{2}\right] \\
& \times \exp \left[-s^{2} R^{d} V_{d}(2 \pi)^{-d} \int d p\left|\hat{g}_{R}(p)\right|^{2} \frac{\zeta}{\mathrm{e}^{\beta|p|^{2}}-\zeta}\right],
\end{aligned}
$$

where $g_{R}$ is defined in (3.9). From the calculations of the previous section, it is trivial that:

$$
\lim _{R \rightarrow \infty} R^{d} V_{d}\left\|g_{R}\right\|^{2}=\lim _{R \rightarrow \infty} \frac{(2 \pi)^{d}}{V_{d}} \int d p\left|\hat{f}\left(\frac{p}{R}\right)\right|^{2} \frac{\left|J_{\frac{d}{2}}(|p|)\right|^{2}}{|p|^{d}}
$$


Since $f$ is assumed to be a Schwartz function, dominated convergence yields immediately

$$
\lim _{R \rightarrow \infty} R^{d} V_{d}\left\|g_{R}\right\|^{2}=2(2 \pi)^{d}|\hat{f}(0)|^{2}
$$

Analogously:

$$
\lim _{R \rightarrow \infty} R^{d} V_{d}(2 \pi)^{-d} \int d p\left|\hat{g}_{R}(p)\right|^{2} \frac{\zeta}{\mathrm{e}^{\beta|p|^{2}}-\zeta}=2|\hat{f}(0)|^{2} \frac{\zeta}{1-\zeta} .
$$

Obviously, the local fluctuations on the normal scale of field operators $\Phi(f)$ for Schwartz functions $f$ with $\hat{f}(0)=0$ do not survive in the limit $R \rightarrow \infty$. The limiting fluctuation observable for each field operator $\Phi(f)$ with $\hat{f}(0)=0$ will consequently be the zero operator, unless one scales the local fluctuations subnormally for these operators.

Proposition 3. For densities $\bar{\rho}<\rho_{c}(\beta), k=0$ mode fluctuations of field operators $\Phi(f)$ with $\hat{f}(0)=0$ exist on a subnormal scale; i.e. with a scaling index $\delta_{f}=$ $-\frac{1}{2 d}$, the characteristic functions are

$$
\begin{aligned}
& \lim _{R \rightarrow \infty} \omega_{\beta, \bar{\rho}}^{\infty}\left(\mathrm{e}^{\mathrm{i} s F_{R}^{-\frac{1}{2 d}}(\Phi(f))}\right) \\
& \quad=\exp \left[-\frac{s^{2}}{2} \frac{V_{d}^{-1+\frac{1}{d}}}{2 \pi} \int d p \frac{|\hat{f}(p)|^{2}}{|p|^{d+1}}\left[\frac{\zeta}{\mathrm{e}^{\beta|p|^{2}}-\zeta}+(2 \pi)^{d}\right]\right] .
\end{aligned}
$$

Proof. For this type of testfunctions

$$
\begin{aligned}
\lim _{R \rightarrow \infty} \omega_{\beta, \bar{\rho}}^{\infty}\left(\mathrm{e}^{\mathrm{i} s F_{R}^{-\frac{1}{2 d}}(\Phi(f))}\right) \\
=\exp \left[-\frac{s^{2}}{4}\left(R^{d} V_{d}\right)^{1+\frac{1}{d}}\left\|g_{R}\right\|^{2}\right] \\
\quad \times \exp \left[-\frac{s^{2}}{2}\left(R^{d} V_{d}\right)^{1+\frac{1}{d}}(2 \pi)^{-d} \int d p\left|\hat{g}_{R}(p)\right|^{2} \frac{\zeta}{\mathrm{e}^{\beta|p|^{2}}-\zeta}\right] .
\end{aligned}
$$

The first factor gives now

$$
\begin{aligned}
& \lim _{R \rightarrow \infty}\left(R^{d} V_{d}\right)^{1+\frac{1}{d}}\left\|g_{R}\right\|^{2} \\
& =\lim _{R \rightarrow \infty}(2 \pi)^{d} V_{d}^{-1+\frac{1}{d}} R \int d p|\hat{f}(p)|^{2} \frac{\left|J_{\frac{d}{2}}(|p| R)\right|^{2}}{|p|^{d}} \\
& \quad=(2 \pi)^{d} V_{d}^{-1+\frac{1}{d}} \lim _{\varepsilon \rightarrow 0+R \rightarrow \infty} \lim _{R} R\left[\int_{0 \leqq|p| \leqq \varepsilon}+\int_{|p| \geqq \varepsilon}\right]|\hat{f}(p)|^{2} \frac{\left|J_{\frac{d}{2}}(|p| R)\right|^{2}}{|p|^{d}} .
\end{aligned}
$$

The integration domain has been split up in two parts in order to simplify the analysis of the limit $R \rightarrow \infty$. 
Introducing the positive function $h$ defined on $\mathbb{R}^{+}$by

$$
h(|p|)=\sqrt{\int d \Omega_{d-1}\left|\hat{f}\left(|p|, \theta_{1}, \ldots, \theta_{d-1}\right)\right|^{2}},
$$

where $\int d \Omega_{d-1}$ is a shorthand notation for the angular integrals and the use of the asymptotic form of the Besselfunction $J_{\frac{d}{2}}(|p| R)$ for large values of $R$,

$$
J_{\frac{d}{2}}(|p| R) \sim \sqrt{\frac{2}{\pi|p| R}} \cos \left[|p| R-\frac{\pi(d+1)}{4}\right]
$$

gives first:

$$
\begin{aligned}
& \lim _{\varepsilon \rightarrow 0+R \rightarrow \infty} \lim _{|p| \geqq \varepsilon} R \int_{|p|} d p \frac{|\hat{f}(p)|^{2}}{|p|^{d}}\left|J_{\frac{d}{2}}(|p| R)\right|^{2} \\
& =\frac{1}{\pi} \lim _{\varepsilon \rightarrow 0+R \rightarrow \infty} \lim _{|p| \geqq \varepsilon} d p \frac{|\hat{f}(p)|^{2}}{|p|^{d+1}} \cos ^{2}\left(|p| R-\frac{\pi(d+1)}{4}\right) \\
& \quad=\frac{1}{2 \pi} \int d p \frac{|\hat{f}(p)|^{2}}{|p|^{d+1}}
\end{aligned}
$$

This is a finite number, since $f$ is a Schwartz function with $\hat{f}(0)=0$ and hence $\int d \Omega_{d-1}\left|\hat{f}\left(|p|, \theta_{1}, \ldots, \theta_{d-1}\right)\right|^{2}$ is at least of order $O\left(|p|^{2}\right)$ around $p=0$.

Secondly, one has

$$
\begin{aligned}
& \lim _{\varepsilon \rightarrow 0+R \rightarrow \infty} \lim _{0} R \int_{0}^{\varepsilon} d|p|\left[\frac{h^{2}(|p|)}{|p|}\left|J_{\frac{d}{2}}(|p| R)\right|^{2}\right] \\
& \sim \lim _{\varepsilon \rightarrow 0+R \rightarrow \infty} \lim _{R \rightarrow \infty} \frac{1}{R} \int_{0}^{\varepsilon R} d|p||p|\left|J_{\frac{d}{2}}(|p|)\right|^{2} \\
& \sim \lim _{\varepsilon \rightarrow 0+R \rightarrow \infty} \lim _{R \rightarrow} \frac{1}{R} O(\varepsilon R) \\
& \quad=0
\end{aligned}
$$

With the same arguments, one proves

$$
\begin{gathered}
\lim _{R \rightarrow \infty}\left(R^{d} V_{d}\right)^{1+\frac{1}{d}}(2 \pi)^{-d} \int d p\left|\hat{g}_{R}(p)\right|^{2} \frac{\zeta}{\mathrm{e}^{\beta|p|^{2}}-\zeta} \\
=V_{d}^{-1+\frac{1}{d}} \frac{1}{2 \pi} \int d p \frac{|\hat{f}(p)|^{2}}{|p|^{d+1}} \frac{\zeta}{\mathrm{e}^{\beta|p|^{2}}-\zeta} \quad \square
\end{gathered}
$$

It is important to note that the limits $F^{\delta_{f}}(\Phi(f))$ can be characterised as welldefined operators on some Hilbert space. To that end one needs a reconstruction theorem. 
Essential ingredients of the reconstruction theorem are the following B-C-H formulae

Lemma 1. One has:

1.

$$
\begin{aligned}
& \lim _{R} \omega_{\beta, \bar{\rho}}^{\infty}\left(\mathrm{e}^{\mathrm{i} F_{R}^{0}\left(\Phi\left(f_{1}\right)\right)} \mathrm{e}^{\mathrm{i} F_{R}^{0}\left(\Phi\left(f_{2}\right)\right)}\right) \\
& \quad=\lim _{R} \omega_{\beta, \bar{\rho}}^{\infty}\left(\mathrm{e}^{\mathrm{i} F_{R}^{0}\left(\Phi\left(f_{1}+f_{2}\right)\right)}\right) \mathrm{e}^{-2 \mathrm{i} \operatorname{Im}\left(\overline{\hat{f}_{1}(0)} \hat{f}_{2}(0)\right)}
\end{aligned}
$$

2.

$$
\begin{aligned}
& \lim _{R} \omega_{\beta, \bar{\rho}}^{\infty}\left(\mathrm{e}^{\mathrm{i} F_{R}^{0}\left(\Phi\left(f_{1}\right)\right)} \mathrm{e}^{\mathrm{i} F_{R}^{-\frac{1}{2 d}}\left(\Phi\left(g_{1}\right)\right)}\right) \\
& \quad=\lim _{R} \omega_{\beta, \bar{\rho}}^{\infty}\left(\mathrm{e}^{\mathrm{i} F_{R}^{0}\left(\Phi\left(f_{1}\right)\right)}\right) \lim _{R} \omega_{\beta, \bar{\rho}}^{\infty}\left(\mathrm{e}^{\mathrm{i} F_{R}^{-\frac{1}{2 d}}\left(\Phi\left(g_{1}\right)\right)}\right) .
\end{aligned}
$$

3.

$$
\begin{aligned}
\lim _{R} \omega_{\beta, \bar{\rho}}^{\infty}\left(\mathrm{e}^{\mathrm{i} F_{R}^{-\frac{1}{2 d}}\left(\Phi\left(g_{1}\right)\right)} \mathrm{e}^{\mathrm{i} F_{R}^{-\frac{1}{2 d}}\left(\Phi\left(g_{2}\right)\right)}\right) \\
=\lim _{R} \omega_{\beta, \bar{\rho}}^{\infty}\left(\mathrm{e}^{\mathrm{i} F_{R}^{-\frac{1}{2 d}}\left(\Phi\left(g_{1}+g_{2}\right)\right)}\right) \\
\quad \times \exp \left[-\frac{\mathrm{i}}{\pi} V_{d}^{\frac{1}{d}-1} \int d p \frac{\operatorname{Im}\left(\overline{\hat{g}_{1}(p)} \hat{g}_{2}(p)\right)}{|p|^{d+1}}\right] .
\end{aligned}
$$

Proof. Working in the GNS representations of the states $\omega_{\beta, \bar{\rho}}^{\infty}$, one has:

$$
\begin{aligned}
\mathrm{e}^{\mathrm{i} F_{R}^{\delta} f}(\Phi(f)) & \mathrm{e}^{\mathrm{i} F_{R}^{\delta_{g}}(\Phi(g))} \\
= & W\left(\left(R^{d} V_{d}\right)^{-\left(\frac{1}{2}+\delta_{f}\right)} \int_{B^{d}(0, R)} d x f^{x}\right) W\left(\left(R^{d} V_{d}\right)^{-\left(\frac{1}{2}+\delta_{y}\right)} \int_{B^{d}(0, R)} d x g^{x}\right) \\
= & W\left(\left(R^{d} V_{d}\right)^{-\frac{1}{2}} \int_{B^{d}(0, R)} d x\left\{\left(R^{d} V_{d}\right)^{-\delta_{f}} f^{x}+\left(R^{d} V_{d}\right)^{-\delta_{y}} g^{x}\right\}\right) \\
& \times \exp \left[-\mathrm{i}\left(R^{d} V_{d}\right)^{-\left(1+\delta_{f}+\delta_{g}\right)} \operatorname{Im}\left(\int_{B^{d}(0, R)} d x f^{x}, \underset{B^{d}(0, R)}{\int} d x g^{x}\right)\right] .
\end{aligned}
$$

The techniques used in the proofs of the central limit theorems, in particular Propositions 2 and 3, are also applicable here and yield:

$$
\begin{gathered}
\operatorname{Im}\left(\left(R^{d} V_{d}\right)^{-\left(\frac{1}{2}+\delta_{f}\right)} \int_{B^{d}(0, R)} d x f^{x},\left(R^{d} V_{d}\right)^{-\left(\frac{1}{2}+\delta_{y}\right)} \int_{B^{d}(0, R)} d x g^{x}\right) \\
=\left(R^{d} V_{d}\right)^{-\left(1+\delta_{f}+\delta_{g}\right)} \int d p \operatorname{Im}(\overline{\hat{f}(p)} \hat{g}(p))\left|\hat{\chi}_{B^{d}(0, R)}(p)\right|^{2} \\
=\frac{\left(R^{d} V_{d}\right)^{\left(\delta_{f}+\delta_{g}\right)}}{V_{d}} \int d p \operatorname{Im}\left(\overline{\hat{f}\left(\frac{p}{R}\right)} \hat{g}\left(\frac{p}{R}\right)\right) \frac{J_{\frac{d}{2}}^{2}(|p|)}{|p|^{d}}
\end{gathered}
$$




$$
\stackrel{R \rightarrow \infty}{\longrightarrow}\left\{\begin{array}{lll}
2 \operatorname{Im}(\overline{\hat{f}(0)} \hat{g}(0)) & \text { iff } \quad \delta_{f}=\delta_{g}=0 \\
0 & \text { iff } \quad \delta_{f} \neq \delta_{g} \\
\frac{V_{d}^{\frac{1}{d}-1}}{\pi} \int d p \frac{\operatorname{Im}(\overline{\hat{f}(p)} \hat{g}(p))}{|p|^{d+1}} & \text { iff } \quad \delta_{f}=\delta_{g}=-\frac{1}{2 d}
\end{array}\right.
$$

The discussion of the situations $\delta_{f}=\delta_{g}=0$ and $\delta_{f}=\delta_{g}=-\frac{1}{2 d}$ is in fact implicitly present in the proofs of the central limit theorems. The situation in which $\delta_{f} \neq \delta_{g}$ remains to be considered; in this case

$$
\begin{aligned}
& \frac{\left(R^{d} V_{d}\right)^{\left(\frac{1}{2 d}\right)}}{V_{d}} \int d p \operatorname{Im}\left(\overline{\hat{f}\left(\frac{p}{R}\right)} \hat{g}\left(\frac{p}{R}\right)\right) \frac{J_{\frac{d}{2}}^{2}(|p|)}{|p|^{d}} \\
& \sim V_{d}^{-1+\frac{1}{2 d}} R^{\frac{1}{2}} R^{-1} \int_{|p| \geqq \varepsilon} d p \operatorname{Im}(\overline{\hat{f}(p)} \hat{g}(p)) \frac{1}{\pi|p|^{d+1}} \\
& \quad+V_{d}^{-1+\frac{1}{2 d}} R^{\frac{1}{2}} \int_{0 \leqq \delta R} d p \operatorname{Im}\left(\overline{\hat{f}\left(\frac{p}{R}\right)} \hat{g}\left(\frac{p}{R}\right)\right) \frac{J_{\frac{d}{2}}^{2}(|p|)}{|p|^{d}} \\
& \stackrel{R \rightarrow \infty}{\longrightarrow} 0 .
\end{aligned}
$$

The limit is zero since either $\hat{f}(0)$ or $\hat{g}(0)$ is zero and the other is not. This leads to an angular integral which is at least of order $O\left(\frac{|p|}{R}\right)$.

It is now also trivial that the distribution of the sum of a normal and a subnormal fluctuation is the product of their distributions:

$$
\begin{gathered}
\lim _{R} \omega_{\beta, \bar{\rho}}^{\infty}\left(W\left(\left(R^{d} V_{d}\right)^{-\frac{1}{2}} \int_{B^{d}(0, R)} d x\left\{\left(R^{d} V_{d}\right)^{-\delta_{f}} f^{x}+\left(R^{d} V_{d}\right)^{-\delta_{y}} g^{x}\right\}\right)\right) \\
=\lim _{R} \omega_{\beta, \bar{\rho}}^{\infty}\left(W\left(\left(R^{d} V_{d}\right)^{-\frac{1}{2}} \int_{B^{d}(0, R)} d x\left(R^{d} V_{d}\right)^{-\delta_{f}} f^{x}\right)\right) \\
\lim _{R} \omega_{\beta, \bar{\rho}}^{\infty}\left(W\left(\left(R^{d} V_{d}\right)^{-\frac{1}{2}} \int_{B^{d}(0, R)} d x\left(R^{d} V_{d}\right)^{-\delta_{y}} g^{x}\right)\right)
\end{gathered}
$$

if either $\delta_{f}$ or $\delta_{g}=-\frac{1}{2 d}$ and the other is 0 .

These B-C-H formulae are very important because they reveal the structure of the algebra of the macroscopic fluctuations of the fields. Consider the symplectic space: $(\mathbb{C} \oplus S, \sigma)$, where $S$ is the linear space of Schwartz functions $f$ with $\hat{f}(0)=$ 0 and where $\sigma=\sigma_{1} \oplus \sigma_{2}$ is defined by

$$
\begin{aligned}
& \sigma_{1}(\alpha, \beta)=4 \operatorname{Im}(\bar{\alpha} \beta) \quad \alpha, \beta \in \mathbb{C}, \\
& \sigma_{2}(f, g)=\frac{2}{\pi} V_{d}^{\frac{1}{d}-1} \int d p \frac{\operatorname{Im}(\overline{\hat{f}(p)} \hat{g}(p))}{|p|^{d+1}} .
\end{aligned}
$$

On this symplectic space $(\mathbb{C} \oplus S, \sigma)$, one can construct the Weyl algebra $W(\mathbb{C} \oplus$ $S, \sigma)$ generated by the Weyl operators $\{W(A) \mid A \in \mathbb{C} \oplus S\}$ which satisfy the product rule

$$
W(A) W(B)=W(A+B) \mathrm{e}^{-\frac{1}{2} \sigma(A, B)} .
$$


In fact, $W(\mathbb{C} \oplus S, \sigma)=W\left(\mathbb{C}, \sigma_{1}\right) \otimes W\left(S, \sigma_{2}\right)$. The reconstruction theorem can now be formulated:

Theorem 3 (Reconstruction theorem for the low density regime). Define for $f \in \mathscr{D}$ and $g \in S$ :

$$
F_{R}(\hat{f}(0) \oplus g)=F_{R}^{0}(\Phi(f))+F_{R}^{-\frac{1}{2 d}}(\Phi(g)) .
$$

The limits $\lim _{R \rightarrow \infty} \omega_{\beta, \bar{\rho}}^{\infty}\left(\mathrm{e}^{\mathrm{i} F_{R}(\hat{f}(0) \oplus g)}\right)$ determine a quasi-free state $\tilde{\omega}=\tilde{\omega}_{1} \otimes \tilde{\omega}_{2}$ on $W(\mathbb{C} \oplus S, \sigma)=W\left(\mathbb{C}, \sigma_{1}\right) \otimes W\left(S, \sigma_{2}\right)$ with generating functional

$$
\begin{aligned}
\tilde{\omega}(W(\hat{f}(0) \oplus g)) & =\tilde{\omega}_{1}\left(W_{1}(\hat{f}(0)) \tilde{\omega}_{2}\left(W_{2}(g)\right)\right. \\
& =\exp \left[-\frac{1}{2} s_{1}(\hat{f}(0), \hat{f}(0))\right] \exp \left[-\frac{1}{2} s_{2}(g, g)\right]
\end{aligned}
$$

with

$$
\begin{aligned}
s_{1}(\hat{f}(0), \hat{f}(0)) & =\lim _{R \rightarrow \infty} \omega_{\beta, \bar{\rho}}^{\infty}\left(\left[F_{R}^{0}(\Phi(f))\right]^{2}\right), \\
s_{2}(g, g) & =\lim _{R \rightarrow \infty} \omega_{\beta, \bar{\rho}}^{\infty}\left(\left[F_{R}^{-\frac{1}{2 d}}(\Phi(g))\right]^{2}\right) .
\end{aligned}
$$

Proof. The previous central limit theorems and the inequality

$$
\begin{aligned}
\frac{1}{4}\left|\sigma\left(\hat{f}_{1}(0) \oplus g_{1}, \hat{f}_{2}(0) \oplus g_{2}\right)\right|^{2} \leqq & \left\{s_{1}\left(\hat{f}_{1}(0), \hat{f}_{1}(0)\right)+s_{2}\left(g_{1}, g_{1}\right)\right\} \\
& \times\left\{s_{1}\left(\hat{f}_{2}(0), \hat{f}_{2}(0)\right)+s_{2}\left(g_{2}, g_{2}\right)\right\}
\end{aligned}
$$

guarantee the existence of the quasi-free state $\tilde{\omega}$. See also reference [10].

This theorem determines the macroscopic fluctuation operators. There corresponds a unique GNS triplet $\left(\tilde{\mathscr{H}}_{1} \otimes \tilde{\mathscr{H}}_{2}, \tilde{\pi}_{1} \otimes \tilde{\pi}_{2}, \tilde{\Omega}_{1} \otimes \tilde{\Omega}_{2}\right)$ to the system $(W(\mathbb{C} \oplus$ $S), \tilde{\omega})$ such that

$$
\tilde{\omega}(W(\hat{f}(0) \oplus g))=\left(\tilde{\Omega}_{1}, \tilde{\pi}_{1}\left(W_{1}(\hat{f}(0))\right) \tilde{\Omega}_{1}\right)\left(\tilde{\Omega}_{2}, \tilde{\pi}_{2}\left(W_{2}(g)\right) \tilde{\Omega}_{2}\right) .
$$

The regularity of a quasi-free state assures the existence of the Bose fields $F^{0}$ and $F^{-\frac{1}{2 d}}$ such that

$$
\tilde{\pi}_{1} \otimes \tilde{\pi}_{2}(W(\hat{f}(0) \oplus g))=\exp \left[\mathrm{i}\left(F^{0}(\hat{f}(0)) \oplus F^{-\frac{1}{2 d}}(g)\right)\right] .
$$

Precisely these Bose field operators $F^{0}(\hat{f}(0))$ and $F^{-\frac{1}{2 d}}(g)$ are called the normal and subnormal fluctuation observables of the field operators. This completes the discussion of the $k=0$ mode fluctuations of the field operators in the low density regime.

\subsection{The high density regime.}

The presence of long-range correlations in the high density regime will lead to the abnormality of the fluctuations of the field operators. In the physics literature, such 
a phenomenon would be characterised by a critical exponent $\eta$ appearing in the asymptotic form of the connected two-point function:

$$
\omega\left(a^{+}(f) \tau_{x} a(f)\right)-\left|\omega\left(a^{+}(f)\right)\right|^{2} \sim O\left(\frac{1}{|x|^{d-2+\eta}}\right) \quad \text { as }|x| \rightarrow \infty
$$

If the connected two point function has this asymptotic behaviour, then it is easily verified that the field fluctuations require a scaling index $\delta_{f}$ which is related to the critical exponent $\eta$ by the following relation:

$$
\eta=2-2 d \delta_{f}
$$

It is standard wisdom that in a free or in a mean field model the critical exponent $\eta$ describing the asymptotic behaviour of the correlations, is zero and hence an abnormal scaling index $\delta_{f}=\frac{1}{d}$ is to be expected. We give a mathematically rigorous proof of this.

Proposition 4. For densities $\bar{\rho} \geqq \rho_{c}(\beta)$, the $k=0$ mode fluctuations of field operators $\Phi(f)$ with $\hat{f}(0) \neq 0$, are abnormal with a scaling index $\delta_{f}=\frac{1}{d}$. In each of the extremal equilibrium states $\omega_{\beta, \bar{\rho}, \theta}^{\infty}(\theta \in[0,2 \pi))$ the limiting distribution of these fluctuations is:

$$
\lim _{R \rightarrow \infty} \omega_{\beta, \bar{\rho}, \theta}^{\infty}\left(\mathrm{e}^{\mathrm{i} s F_{R}^{\frac{1}{d}}(\Phi(f))}\right)=\exp \left[-s^{2} V_{d}^{-\frac{2}{d \beta}} d|\hat{f}(0)|^{2} \int_{0}^{\infty} d x \frac{J_{\frac{d}{2}}^{2}(x)}{x^{3}}\right] .
$$

Proof. Consider the characteristic function of the field fluctuations

$$
\begin{aligned}
\omega_{\beta, \bar{\rho}, \theta}^{\infty}\left(\mathrm{e}^{\mathrm{i} s F_{R}^{\frac{1}{d}}(\Phi(f))}\right)= & \exp \left[-\frac{s^{2}}{2} R^{d-2} V_{d}^{1-\frac{2}{d}}\left\|g_{R}\right\|\right] \\
& \times \exp \left[-s^{2} R^{d-2} V_{d}^{1-\frac{2}{d}}(2 \pi)^{-d} \int d p \frac{\left|\hat{g}_{R}(p)\right|^{2}}{\mathrm{e}^{\beta|p|^{2}}-1}\right]
\end{aligned}
$$

Concerning the first factor of (4.17), it has been proved in Proposition 2 that

$$
\lim _{R \rightarrow \infty} R^{d} V_{d}\left\|g_{R}\right\|^{2}=2(2 \pi)^{d}|\hat{f}(0)|^{2}
$$

Consequently

$$
R^{d-2} V_{d}^{1-\frac{2}{d}}\left\|g_{R}\right\| \stackrel{R \rightarrow \infty}{\longrightarrow} 0
$$

due to the abnormal scaling of the fluctuations of the field operators.

It is important to note that this will imply the abelian character of the macroscopic fluctuation observables of these field operators.

The extra volume factor $R^{-1} V_{d}^{-\frac{1}{d}}$ in the scaling of the local fluctuations is required to compensate the diverging contribution of the factor $\frac{1}{\mathrm{e}^{\beta|p|^{2}}-1}$ in the 
integrand of the second factor in (4.17). Using again the function $h$ defined in (4.11), one writes:

$$
\begin{aligned}
& \lim _{R \rightarrow \infty} V_{d}^{1-\frac{2}{d}} R^{d-2}(2 \pi)^{-d} \int d p \frac{\left|\hat{g}_{R}(p)\right|^{2}}{\mathrm{e}^{\beta|p|^{2}}-1} \\
& =\lim _{\varepsilon \rightarrow 0+} \lim _{R} V_{d}^{-1-\frac{2}{d}} R^{-2}\left[\int_{0}^{\varepsilon R} d x+\int_{\varepsilon R}^{\infty} d x\right] \frac{\left|J_{\frac{d}{2}}(x)\right|^{2} h^{2}\left(\frac{x}{R}\right)}{x\left(\mathrm{e}^{\beta \frac{x^{2}}{R^{2}}}-1\right)}
\end{aligned}
$$

By dominated convergence:

$$
\lim _{R} V_{d}^{-1-\frac{2}{d}} R^{-2} \int_{\varepsilon R}^{\infty} d x \frac{\left|J_{\frac{d}{2}}(x)\right|^{2} h^{2}\left(\frac{x}{R}\right)}{x\left(\mathrm{e}^{\beta \frac{x^{2}}{R^{2}}}-1\right)}=0
$$

The other term can be rewritten as

$$
\begin{gathered}
V_{d}^{-1-\frac{2}{d}} R^{-2} \int_{0}^{\varepsilon R} d x \frac{\left|J_{\frac{d}{2}}(x)\right|^{2}\left\{h^{2}\left(\frac{x}{R}\right)-|\hat{f}(0)|^{2}\right\}}{x\left(\mathrm{e}^{\beta \frac{x^{2}}{R^{2}}}-1\right)} \\
+V_{d}^{-1-\frac{2}{d}} R^{-2} \int_{0}^{\varepsilon R} d x \frac{\left|J_{\frac{d}{2}}(x)\right|^{2}|\hat{f}(0)|^{2} \Phi_{d-1}}{x\left(\mathrm{e}^{\beta \frac{x^{2}}{R^{2}}}-1\right)}
\end{gathered}
$$

The limits of the previous expressions can be determined using the inequalities

$$
\int_{0}^{\varepsilon R} d x \frac{J_{\frac{d}{2}}^{2}(x)}{x \beta \frac{x^{2}}{R^{2}} 1} \geqq \int_{0}^{\varepsilon R} d x \frac{J_{\frac{d}{2}}^{2}(x)}{x \beta \frac{x^{2}}{R^{2}} \frac{\mathrm{e}^{\beta \frac{x^{2}}{R^{2}}-1}}{\beta \frac{x^{2}}{R^{2}}}} \geqq \int_{0}^{\varepsilon R} d x \frac{J_{\frac{d}{2}}^{2}(x)}{x \beta \frac{x^{2}}{R^{2}} \frac{\mathrm{e}^{\beta \varepsilon^{2}-1}}{\beta \varepsilon^{2}}} .
$$

The conclusion is that

$$
\begin{aligned}
& \lim _{\varepsilon \rightarrow 0+R \rightarrow \infty} \lim _{d} V^{-1-\frac{2}{d}} R^{-2} \int_{0}^{\varepsilon R} d x \frac{J_{\frac{d}{2}}^{2}(x)|\hat{f}(0)|^{2} \Phi_{d-1}}{x\left(\mathrm{e}^{\beta \frac{x^{2}}{R^{2}}}-1\right)} \\
& =V_{d}^{-\frac{2}{d}} \frac{d}{\beta}|\hat{f}(0)|^{2} \int_{0}^{\infty} d x \frac{J_{\frac{d}{2}}^{2}(x)}{x^{3}}
\end{aligned}
$$

and that

$$
\lim _{\varepsilon \rightarrow 0+R \rightarrow \infty} \lim _{d} V^{-1-\frac{2}{d}} R^{-2} \int_{0}^{\varepsilon R} d x \frac{J_{\frac{d}{2}}^{2}(x)\left\{h^{2}\left(\frac{x}{R}\right)-|\hat{f}(0)|^{2}\right\}}{x\left(\mathrm{e}^{\beta \frac{x^{2}}{R^{2}}}-1\right)}=0 .
$$

Following the analysis of the low density regime, the next question is of course: what about the field operators for which $\hat{f}(0)=0$. The answer is a little more complicated for the high density regime, because there are two types of Schwartz functions to be considered. For a first class of Schwartz functions $f$ the $\delta_{f}=0$ and for a second class of Schwartz functions $g, \delta_{g}$ is equal to $-\frac{1}{2 d}$. It seems that for 
the second class the appearance of a Bose condensate has no influence at all on the behaviour of the corresponding field fluctuations.

Let us begin with the class of Schwartz functions $f$ with $\hat{f}(0)=0$ and which have moreover the property that for some $i \in\{1, \ldots, d\}:\left.\frac{\partial \hat{f}}{\partial p_{i}}\right|_{p=0} \neq 0$. In this case the function $h^{2}(|p|)(4.11)$ associated to $f$ is of order $O\left(|p|^{2}\right)$; i.e. $\left.\frac{d h}{d|p|}\right|_{|p|=0} \neq 0$. For this kind of functions $f$, the following proposition holds:

Proposition 5. For densities $\bar{\rho} \geqq \rho_{c}(\beta)$, the $k=0$ mode fluctuations of the field operators with $\hat{f}(0)=0$ and the corresponding function $h^{2}(|p|)$ of order $O\left(|p|^{2}\right)$ have no scaling index or equivalently $\delta_{f}=0$. In each of the extremal equilibrium states $\omega_{\beta, \bar{\rho}, 0}^{\infty}$ with $\theta \in[0,2 \pi)$ the limiting distributions of the field fluctuations are:

$$
\lim _{R \rightarrow \infty}\left(\mathrm{e}^{\mathrm{i} s F_{R}^{0}(\Phi(f))}\right)=\exp \left[-s^{2} \frac{2\left(h^{\prime}(0)\right)^{2}}{d V_{d} \beta}\right] .
$$

Proof.

$$
\begin{aligned}
\omega_{\beta, \bar{\rho}, \theta}^{\infty}\left(\mathrm{e}^{\mathrm{i} s F_{R}^{0}(\Phi(f))}\right)= & \exp \left[-\frac{s^{2}}{2} R^{d} V_{d}\left\|g_{R}\right\|\right] \\
& \times \exp \left[-s^{2} R^{d} V_{d}(2 \pi)^{-d} \int d p \frac{\left|\hat{g}_{R}(p)\right|^{2}}{\mathrm{e}^{\beta|p|^{2}}-1}\right] .
\end{aligned}
$$

From the Proof of Proposition 2 we know that

$$
\lim _{R \rightarrow \infty} R^{d} V_{d}\left\|g_{R}\right\|^{2}=0 \text {. }
$$

Again, the limiting fluctuation observables will be abelian.

To discuss the second exponential factor, we rewrite

$$
\begin{aligned}
& \lim _{R \rightarrow \infty} R^{d} V_{d}(2 \pi)^{-d} \int d p \frac{\left|\hat{g}_{R}(p)\right|^{2}}{\mathrm{e}^{\beta|p|^{2}}-1} \\
& \quad=\lim _{\varepsilon \rightarrow 0+R \rightarrow \infty} \lim _{d} V_{0}^{-1}\left[\int_{0}^{\varepsilon R} d x+\int_{\varepsilon R}^{\infty} d x\right] \frac{J_{\frac{d}{2}}^{2}(x) h^{2}\left(\frac{x}{R}\right)}{x\left(\mathrm{e}^{\beta \frac{x^{2}}{R^{2}}}-1\right)} .
\end{aligned}
$$

By dominated convergence

$$
\int_{\varepsilon}^{\infty} d x \frac{J_{\frac{d}{2}}^{2}(x R) h^{2}(x)}{x\left(\mathrm{e}^{\beta x^{2}}-1\right)} \stackrel{R \rightarrow \infty}{\rightarrow} 0 .
$$

For the other integral, we use the asymptotic behaviour of $h^{2}(x)$ for small $x$ and obtain

$$
\begin{aligned}
V_{d}^{-1} \int_{0}^{\varepsilon R} d x \frac{J_{\frac{d}{2}}^{2}(x) h^{2}\left(\frac{x}{R}\right)}{x\left(\mathrm{e}^{\beta \frac{x^{2}}{R^{2}}}-1\right)} & \sim V_{d}^{-1} \int_{0}^{\varepsilon R} d x \frac{J_{\frac{d}{2}}^{2}(x)\left(h^{\prime}(0)\right)^{2} \frac{x^{2}}{R^{2}}}{x \beta \frac{x^{2}}{R^{2}}} \\
& \stackrel{R \rightarrow \infty}{\longrightarrow} \frac{2\left(h^{\prime}(0)\right)^{2}}{d V_{d} \beta} .
\end{aligned}
$$


The other class of test functions consists of the Schwartz functions with $\hat{f}(0)=$ 0 as well as all partial derivatives of $f$ in the origin $\left.\frac{\partial f}{\partial p_{i}}\right|_{p=0}=0 ; i \in\{1, \ldots, d\}$. This implies that $h^{2}(x) \sim O\left(x^{2 n}\right)$ with $n \geqq 2$.

Proposition 6. For densities $\bar{\rho} \geqq \rho_{c}(\beta)$, the $k=0$ mode fluctuations of the field operators with $\hat{f}(0)=0$ and corresponding $h^{2}(|p|)$ of order $O\left(|p|^{2 n}\right)$ with $n \geqq 2$ have a scaling index $\delta_{f}=-\frac{1}{2 d}$. In every extremal equilibrium state $\omega_{\beta, \bar{\rho}, \theta}^{\infty}$ with $\bar{\theta} \in$ $[0,2 \pi)$, the limiting distributions of the field operator fluctuations are

$$
\begin{aligned}
& \lim _{R \rightarrow \infty} \omega_{\beta, \bar{\rho}, \theta}^{\infty}\left(\mathrm{e}^{\mathrm{i} s F_{R}^{-\frac{1}{2 d}}(\Phi(f))}\right) \\
& \quad=\exp \left[-\frac{s^{2}}{2} \frac{V_{d}^{-1+\frac{1}{d}}}{2 \pi} \int d p \frac{|\hat{f}(p)|^{2}}{|p|^{d+1}}\left[\frac{1}{\mathrm{e}^{\beta|p|^{2}}-1}+(2 \pi)^{d}\right]\right] .
\end{aligned}
$$

Proof. In the Proof of Proposition 3, it has been shown that

$$
\lim _{R \rightarrow \infty}\left(R^{d} V_{d}\right)^{1+\frac{1}{d}}\left\|g_{R}\right\|^{2}=V_{d}^{-1+\frac{1}{d}}(2 \pi)^{d-1} \int \frac{|\hat{f}(p)|^{2}}{|p|^{d+1}}
$$

This means that this is the only kind of testfunctions $f$ for which the quantum nature of the limiting fluctuation observables of the field operators $\Phi(f)$ is preserved in the high density regime.

Moreover,

$$
\begin{aligned}
& \lim _{R \rightarrow \infty}\left(R^{d} V_{d}\right)^{1+\frac{1}{d}}(2 \pi)^{-d} \int d p \frac{\left|\hat{g}_{R}(p)\right|^{2}}{\mathrm{e}^{\beta|p|^{2}}-1} \\
& \quad=\lim _{\varepsilon \rightarrow 0+} \lim _{R \rightarrow \infty} V_{d}^{-1+\frac{1}{d}} R\left[\int_{0}^{\varepsilon R} d x+\int_{\varepsilon R}^{\infty} d x\right] \frac{J_{\frac{d}{2}}^{2}(x) h^{2}\left(\frac{x}{R}\right)}{x\left(\mathrm{e}^{\beta \frac{x^{2}}{R^{2}}}-1\right)} .
\end{aligned}
$$

Treating again the two terms separately

$$
\begin{aligned}
& \lim _{R \rightarrow \infty} V_{d}^{-1+\frac{1}{d}} R \int_{0}^{\varepsilon R} d x \frac{J_{\frac{d}{2}}^{2}(x) h^{2}\left(\frac{x}{R}\right)}{x\left(\mathrm{e}^{\beta \frac{x^{2}}{R^{2}}}-1\right)} \\
& \quad \sim \lim _{R \rightarrow \infty} V_{d}^{-1+\frac{1}{d}} R \int_{0}^{\varepsilon R} d x \frac{J_{\frac{d}{2}}^{2}(x) O\left(\left(\frac{x}{R}\right)^{2 n-2}\right)}{x} \\
& \quad \sim \lim _{R \rightarrow \infty} V_{d}^{-1+\frac{1}{d}} R^{3-2 n} O\left(\varepsilon R^{2 n-3}\right) \\
& \stackrel{\varepsilon \rightarrow 0+}{\longrightarrow} 0
\end{aligned}
$$


and

$$
\begin{aligned}
& \lim _{\varepsilon \rightarrow 0+} \lim _{R \rightarrow \infty} V_{d}^{-1+\frac{1}{d}} R \int_{\varepsilon R}^{\infty} d x \frac{J_{\frac{d}{2}}^{2}(x) h^{2}\left(\frac{x}{R}\right)}{x\left(\mathrm{e}^{\beta \frac{x^{2}}{R^{2}}}-1\right)} \\
& \quad=\lim _{\varepsilon \rightarrow 0+} \lim _{R \rightarrow \infty} V_{d}^{-1+\frac{1}{d}} \int_{\varepsilon}^{\infty} d x \frac{h^{2}(x)\left[1+\cos \left(2 x R-\frac{\pi(d+1)}{2}\right)\right]}{x^{2}\left(\mathrm{e}^{\beta x^{2}}-1\right)} \\
& \quad=V_{d}^{-1+\frac{1}{d}} \int_{0}^{\infty} d x \frac{h^{2}(x)}{x^{2}\left(\mathrm{e}^{\beta x^{2}}-1\right)},
\end{aligned}
$$

which is a finite number, due to the asymptotic character of $h^{2}$ around $x=0$.

These three central limit theorems (for scaling indices $\frac{1}{d}, 0$ and $-\frac{1}{2 d}$ ) are basic for the reconstruction theorem characterising the limiting observables $F^{\delta}(\Phi(f))$ in the high density regime as well defined operators on some Hilbert space.

Consider again in the G.N.S. representations of the states $\omega_{\beta, \bar{\rho}, \theta}^{\infty}$ the product of two exponentiated to local fluctuations:

$$
\begin{aligned}
& \mathrm{e}^{\mathrm{i} F_{R}^{\delta} f}(\Phi(f)) \mathrm{e}^{\mathrm{i} F_{R}^{\delta}(\Phi(g))} \\
& =W\left(\left(R^{d} V_{d}\right)^{-\left(\frac{1}{2}+\delta_{f}\right)} \int_{B^{d}(0, R)} d x f^{x}\right) W\left(\left(R^{d} V_{d}\right)^{-\left(\frac{1}{2}+\delta_{g}\right)} \int_{B^{d}(0, R)} d x g^{x}\right) \\
& =W\left(\left(R^{d} V_{d}\right)^{-\frac{1}{2}} \int_{B^{d}(0, R)} d x\left\{\left(R^{d} V_{d}\right)^{-\delta_{f}} f^{x}+\left(R^{d} V_{d}\right)^{-\delta_{g}} g^{x}\right\}\right) \\
& \mathrm{e}^{-\mathrm{i}\left(R^{d} V_{d}\right)^{-\left(1+\delta f^{+\delta} \delta_{g}\right)} \operatorname{Im}}\left(\int_{B^{d}(0, R)} d x f^{x}, \int_{B^{d}(0, R)} d x g^{x}\right) .
\end{aligned}
$$

On the basis of Schwarz inequality and the central limit theorems, it is easy to prove that

$$
\lim _{R \rightarrow \infty}\left(R^{d} V_{d}\right)^{-\left(\frac{1}{2}+\delta_{f}+\delta_{g}\right)}\left(\int_{B^{d}(0, R)} d x f^{x}, \int_{B^{d}(0, R)} d x g^{x}\right)=0
$$

for every possible combination of the scaling indices $\delta_{f}$ and $\delta_{g}$, except for the one in which $\delta_{f}$ and $\delta_{g}$ are simultaneously equal to $-\frac{1}{2 d}$. For this last possibility, one knows from Proposition 3 that

$$
\begin{aligned}
& \lim _{R \rightarrow \infty}\left(R^{d} V_{d}\right)^{-\left(\frac{1}{2}+\delta_{f}+\delta_{y}\right)}\left(\int_{B^{d}(0, R)} d x f^{x}, \int_{B^{d}(0, R)} d x g^{x}\right) \\
& =\frac{V_{d}^{\frac{1}{d}-1}}{\pi} \int d p \frac{\operatorname{Im} \overline{\hat{f}(p)} \hat{g}(p)}{|p|^{d+1}} .
\end{aligned}
$$


These considerations lead to the following B-C-H relations summarised in the following lemma:

\section{Lemma 2.}

$$
\begin{aligned}
& \lim _{R \rightarrow \infty} \omega_{\beta, \bar{\rho}, \theta}^{\infty}\left(\mathrm{e}^{\mathrm{i} F_{R}^{\delta} f}(\Phi(f)) \mathrm{e}^{1 F_{R}^{\delta_{g}}(\Phi(g))}\right) \\
& =\lim _{R \rightarrow \infty} \omega_{\beta, \bar{\rho}, \theta}^{\infty}\left(W\left(\left(R^{d} V_{d}\right)^{-\frac{1}{2}} \int_{B^{d}(0, R)} d x\left\{\left(R^{d} V_{d}\right)^{-\delta} f f^{x}+\left(R^{d} V_{d}\right)^{-\delta_{g}} g^{x}\right\}\right)\right) \\
& \quad \times \exp \left[-\frac{\mathrm{i}}{\pi} \delta_{\delta_{f},-\frac{1}{2 d}} \delta_{\delta_{g},-\frac{1}{2 d}} V_{d}^{\frac{1}{d}-1} \int d p \frac{\operatorname{Im} \overline{\hat{f}(p)} \hat{g}(p)}{|p|^{d+1}}\right],
\end{aligned}
$$

where $\delta_{\delta_{f},-\frac{1}{2 d}}$ is equal to 1 if $\delta_{f}=-\frac{1}{2 d}$ and 0 otherwise.

Proof. The considerations preceding the formulation of the lemma reduce its proof to proving the existence of the limits

$$
\lim _{R \rightarrow \infty} \omega_{\beta, \bar{\rho}, \theta}^{\infty}\left(W\left(\left(R^{d} V_{d}\right)^{-\frac{1}{2}} \int_{B^{d}(0, R)} d x\left\{\left(R^{d} V_{d}\right)^{-\delta_{f}} f^{x}+\left(R^{d} V_{d}\right)^{-\delta_{g}} g^{x}\right\}\right)\right)
$$

For $\delta_{f}=\delta_{g}$, this was exactly the subject of the central limit theorems for the high density regime. If $\delta_{f} \neq \delta_{g}$, this characteristic function is equal to

$$
\begin{aligned}
& =\lim _{R \rightarrow \infty} \exp \left[-V_{d}^{-1-2 \delta_{f}} R^{-2 d \delta_{f}} \int d p \frac{|\hat{f}(p)|^{2} J_{\frac{d}{2}}^{2}(|p| R)}{|p|^{d}\left(\mathrm{e}^{\beta|p|^{2}}-1\right)}\right] \\
& \quad \times \lim _{R \rightarrow \infty} \exp \left[-V_{d}^{-1-2 \delta_{g}} R^{-2 d \delta_{y}} \int d p \frac{|\hat{g}(p)|^{2} J_{\frac{d}{2}}^{2}(|p| R)}{|p|^{d}\left(\mathrm{e}^{\beta|p|^{2}}-1\right)}\right] \\
& \quad \times \lim _{R \rightarrow \infty} \exp \left[-V_{d}^{-1-\delta_{f}-\delta_{g}} R^{-d\left(\delta_{f}+\delta_{g}\right)} \int d p \frac{2 \operatorname{Re}(\overline{\hat{f}(p)} \hat{g}(p)) J_{\frac{d}{2}}^{2}(|p| R)}{|p|^{d}\left(\mathrm{e}^{\beta|p|^{2}}-1\right)}\right] .
\end{aligned}
$$

The central limit theorems guarantee again that the first two factors are well defined for any $\delta_{f}$ and $\delta_{g}$. To discuss the last factor, one has to distinguish the following cases: with the shorthand notation

$$
I(f, g)(|p|)=\int d \Omega_{d-1} \operatorname{Re}\left(\overline{\hat{f}\left(|p|, \theta_{1}, \ldots\right)} \hat{g}\left(|p|, \theta_{1}, \ldots\right)\right)
$$


one has

1. First, if $\left\{\begin{array}{l}\delta_{f}=\frac{1}{d} \\ \delta_{g}=0\end{array}\right.$ (or vice versa), then

$$
\begin{aligned}
\lim _{R \rightarrow \infty} & \frac{1}{R} \int d p \frac{\operatorname{Re}(\overline{\hat{f}(p)} \hat{g}(p)) J_{\frac{d}{2}}^{2}(|p| R)}{|p|^{d}\left(\mathrm{e}^{\beta|p|^{2}}-1\right)} \\
= & \lim _{\varepsilon \rightarrow 0+R \rightarrow \infty} \lim _{R} \frac{1}{R} \int_{0}^{\varepsilon R} d|p| \frac{I(f, g)\left(\frac{|p|}{R}\right) J_{\frac{d}{2}}^{2}(|p|)}{|p|\left(\mathrm{e}^{\beta \frac{|p|^{2}}{R^{2}}}-1\right)} \\
& +\lim _{\varepsilon \rightarrow 0+R \rightarrow \infty} \lim _{\varepsilon} \frac{1}{R} \int_{\varepsilon}^{\infty} d|p| \frac{I(f, g)(|p|) J_{\frac{d}{2}}^{2}(|p| R)}{|p|\left(\mathrm{e}^{\beta|p|^{2}}-1\right)} \\
= & \lim _{\varepsilon \rightarrow 0+R \rightarrow \infty} \lim _{R \rightarrow} R^{-2} \int_{0}^{\varepsilon R} d|p| \frac{\left.\mid \frac{|p|}{R}\right) J_{\frac{d}{2}}^{2}(|p|)}{|p| \beta O\left(\frac{|p|^{2}}{R^{2}}\right)}+\lim _{\varepsilon \rightarrow 0+} \lim _{R \rightarrow \infty} O\left(R^{-2}\right) \\
= & \left.\frac{1}{\beta} \frac{d}{d|p|} \int d \Omega_{d-1} \operatorname{Re}\left(\overline{\hat{f}(0)} \hat{g}\left(|p|, \theta_{1}, \ldots\right)\right)\right|_{|p|=0} \int_{0}^{\infty} d x \frac{J_{\frac{d}{2}}^{2}(x)}{x^{2}} .
\end{aligned}
$$

2. On the other hand, if $\left\{\begin{array}{l}\delta_{f}=\frac{1}{d} \\ \delta_{y}=-\frac{1}{2 d}\end{array}\right.$ (or vice versa), then

$$
\begin{aligned}
& \operatorname{Re}(\overline{\hat{f}(p)} \hat{g}(p)) J_{\frac{d}{2}}^{2}(|p| R) \\
& \lim _{R \rightarrow \infty} R^{-d\left(\frac{1}{d}-\frac{1}{2 d}\right)} \int d p \frac{\operatorname{Re}(\hat{f}(p) g(p)) \int_{\frac{d}{2}}^{2}(|p| R)}{|p|^{d}\left(\mathrm{e}^{\beta|p|^{2}}-1\right)} \\
& =\lim _{\varepsilon \rightarrow 0+R \rightarrow \infty} \lim ^{-\frac{1}{2}} \int_{0}^{\varepsilon R} d|p| \frac{I(f, g)\left(\frac{|p|}{R}\right) J_{\frac{d}{2}}^{2}(|p|)}{|p|\left(\mathrm{e}^{\beta \frac{|p|^{2}}{R^{2}}}-1\right)} \\
& +\lim _{\varepsilon \rightarrow 0+R \rightarrow \infty} \lim _{R \rightarrow} R^{-\frac{1}{2}} \int_{\varepsilon}^{\infty} d|p| \frac{I(f, g)(|p|) J_{\frac{d}{2}}^{2}(|p| R)}{|p|\left(\mathrm{e}^{\beta|p|^{2}}-1\right)} \\
& =\lim _{\varepsilon \rightarrow 0+R \rightarrow \infty} \lim R^{-\frac{1}{2}} \int_{0}^{\varepsilon R} d|p| \frac{O\left(\frac{|p|^{n}}{R^{n}}\right) J_{\frac{d}{2}}^{2}(|p|)}{|p| \beta O\left(\frac{|p|^{2}}{R^{2}}\right)} \quad(\text { for } n \geqq 2) \\
& \left.+\lim _{\varepsilon \rightarrow 0+R \rightarrow \infty} \lim _{\varepsilon \rightarrow R^{-\frac{3}{2}}}\right) \\
& =\lim _{\varepsilon \rightarrow 0+R \rightarrow \infty} \lim _{R \rightarrow \infty} O\left(R^{-\frac{1}{2}+2-n+n-3}\right) \\
& =0 \text {. }
\end{aligned}
$$


3. Finally, if $\left\{\begin{array}{l}\delta_{f}=0 \\ \delta_{g}=-\frac{1}{2 d}\end{array}\right.$ (or vice versa), then

$$
\begin{aligned}
& \lim _{R \rightarrow \infty} R^{-d}\left(-\frac{1}{2 d}\right) \int d p \frac{\operatorname{Re}(\overline{\hat{f}(p)} \hat{g}(p)) J_{\frac{d}{2}}^{2}(|p| R)}{|p|^{d}\left(\mathrm{e}^{\beta|p|^{2}}-1\right)} \\
& =\lim _{\varepsilon \rightarrow 0+} \lim _{R \rightarrow \infty} R^{\frac{1}{2}} \int_{0}^{\varepsilon R} d|p| \frac{I(f, g)\left(\frac{|p|}{R}\right) J_{\frac{d}{2}}^{2}(|p|)}{|p|\left(\mathrm{e}^{\beta \frac{|p|^{2}}{R^{2}}}-1\right)} \\
& +\lim _{\varepsilon \rightarrow 0+R \rightarrow \infty} \lim _{R} R^{\frac{1}{2}} \int_{\varepsilon}^{\infty} d|p| \frac{I(f, g)(|p|) J_{\frac{d}{2}}^{2}(|p| R)}{|p|\left(\mathrm{e}^{\beta|p|^{2}}-1\right)} \\
& =\lim _{\varepsilon \rightarrow 0+R \rightarrow \infty} \lim _{R} R^{\frac{1}{2}} \int_{0}^{\varepsilon R} d|p| \frac{O\left(\frac{|p|^{1+n}}{R^{1+n}}\right) J_{\frac{d}{2}}^{2}(|p|)}{|p| \beta O\left(\frac{|p|^{2}}{R^{2}}\right)} \quad(\text { for } n \geqq 2) \\
& +\lim _{\varepsilon \rightarrow 0+} \lim _{R \rightarrow \infty} O\left(R^{\frac{1}{2}-1}\right) \\
& =\lim _{\varepsilon \rightarrow 0+} \lim _{R \rightarrow \infty} O\left(R^{\frac{1}{2}+1-n+n-2}\right) \\
& =0 \text {. }
\end{aligned}
$$

In the calculations above, we used the asymptotic behaviour of the integrals

$$
I(f, g)(|p|)=\int d \Omega_{d-1} \operatorname{Re}\left(\overline{\hat{f}\left(|p|, \theta_{1}, \ldots\right)} \hat{g}\left(|p|, \theta_{1}, \ldots\right)\right)
$$

for small $|p|$ and the asymptotic form of the Besselfunction $J_{\frac{d}{2}}(|p| R)$ for large values of $R$

$$
J_{\frac{d}{2}}(|p| R) \sim \sqrt{\frac{2}{\pi|p| R}} \cos \left[|p| R-\frac{\pi(d+1)}{4}\right] .
$$

It follows from this result that the relevant symplectic space for the CCR algebra of macroscopic field fluctuations in the high density regime of a Bose gas will be $\left(\mathbb{C} \oplus \mathbb{R} \oplus S^{\prime}, \sigma\right)$. The space $S^{\prime}$ is the linear space of Schwartz functions $f$ with $\hat{f}$ analytic in a neighbourhood of $p=0$, satisfying moreover

$$
\begin{gathered}
\hat{f}(0)=0 \\
\left.\frac{\partial \hat{f}}{\partial p_{i}}\right|_{p=0}=0 \quad \forall i \in\{1, \ldots, d\} .
\end{gathered}
$$

The symplectic form will be $\sigma=0 \oplus 0 \oplus \sigma_{2}$ where

$$
\sigma_{2}(f, g)=\frac{2}{\pi} V_{d}^{\frac{1}{d}-1} \int d p \frac{\operatorname{Im}(\overline{\hat{f}(p)} \hat{g}(p))}{|p|^{d+1}}
$$


We construct on this symplectic space the Weyl algebra $W\left(\mathbb{C} \oplus \mathbb{R} \oplus S^{\prime}, \sigma\right)$ generated by the Weyl operators $\left\{W(A) \mid A \in \mathbb{R} \oplus \mathbb{R} \oplus S^{\prime}\right\}$ satisfying the product rule

$$
W(A) W(B)=W(A+B) \mathrm{e}^{-\frac{\mathrm{i}}{2} \sigma(A, B)} .
$$

It is trivial to see that

$$
W\left(\mathbb{C} \oplus \mathbb{R} \oplus S^{\prime}, \sigma\right)=W(\mathbb{C}, 0) \otimes W(\mathbb{R}, 0) \otimes W\left(S^{\prime}, \sigma_{2}\right) .
$$

The reconstruction theorem for the high density regime looks as follows:

Theorem 4 (Reconstruction theorem for the high density regime). Define for $f_{1}, f_{2} \in \mathscr{D}$ with $\hat{f}_{2}(0)=0$ and $\left.x\left(f_{2}\right) \equiv \frac{d}{d|p|} \sqrt{\int d \Omega_{d-1}\left|\hat{f}_{2}\right|^{2}}\right|_{|p|=0}$ and for $f_{3} \in S^{\prime}$,

$$
F_{R}\left(\hat{f}_{1}(0) \oplus x\left(f_{2}\right) \oplus f_{3}\right)=F_{R}^{\frac{1}{d}}\left(\Phi\left(f_{1}\right)\right)+F_{R}^{0}\left(\Phi\left(f_{2}\right)\right)+F_{R}^{-\frac{1}{2 d}}\left(\Phi\left(f_{3}\right)\right) .
$$

The limits

$$
\lim _{R \rightarrow \infty} \omega_{\beta, \bar{\rho}, \theta}^{\infty}\left(\exp \left[\mathrm{i} F_{R}\left(\hat{f}_{1}(0) \oplus x\left(f_{2}\right) \oplus f_{3}\right)\right]\right)
$$

determine then a quasi-free state $\tilde{\omega}=\tilde{\omega}_{0} \otimes \tilde{\omega}_{\sigma_{2}}$ on $(W(\mathbb{C}, 0) \otimes W(\mathbb{R}, 0)) \otimes$ $W\left(S^{\prime}, \sigma\right)$ by

$$
\begin{aligned}
\tilde{\omega}( & \left.W\left(\hat{f}_{1}(0) \oplus x\left(f_{2}\right) \oplus f_{3}\right)\right) \\
& =\tilde{\omega}_{0}\left(W\left(\hat{f}_{1}(0)\right) \otimes W\left(x\left(f_{2}\right)\right)\right) \tilde{\omega}_{\sigma_{2}}\left(W\left(f_{3}\right)\right) \\
& =\exp \left[-\frac{1}{2} s_{0}\left(\hat{f}_{1}(0) \oplus x\left(f_{2}\right), \hat{f}_{1}(0) \oplus x\left(f_{2}\right)\right)\right] \exp \left[-\frac{1}{2} s_{\sigma_{2}}\left(f_{3}, f_{3}\right)\right]
\end{aligned}
$$

with

$$
\begin{aligned}
s_{0}\left(\hat{f}_{1}(0) \oplus x\left(f_{2}\right), \hat{f}_{1}(0) \oplus x\left(f_{2}\right)\right) & =\lim _{R \rightarrow \infty} \omega_{\beta, \bar{\rho}, \theta}^{\infty}\left(\left[F_{R}^{\frac{1}{d}}\left(\Phi\left(f_{1}\right)\right)+F_{R}^{0}\left(\Phi\left(f_{2}\right)\right)\right]^{2}\right) \\
s_{\sigma_{2}}\left(f_{3}, f_{3}\right) & =\lim _{R \rightarrow \infty} \omega_{\beta, \bar{\rho}, \theta}^{\infty}\left(\left[F_{R}^{-\frac{1}{2 d}}\left(\Phi\left(f_{3}\right)\right)\right]^{2}\right) .
\end{aligned}
$$

Proof. The central limit theorems and the inequality

$$
\frac{1}{4}\left|\sigma_{2}\left(f_{3}, g_{3}\right)\right|^{2} \leqq s_{\sigma_{2}}\left(f_{3}, f_{3}\right) s_{\sigma_{2}}\left(g_{3}, g_{3}\right)
$$

guarantee the existence of the quasi-free state $\tilde{\omega}([10])$.

One can now determine the macroscopic fluctuation operators for the high density regime. In the GNS triplet $\left(\tilde{\mathscr{H}}_{0} \otimes \tilde{\mathscr{H}}_{2}, \tilde{\pi}_{0} \otimes \tilde{\pi}_{2}, \tilde{\Omega}_{0} \otimes \tilde{\Omega}_{2}\right)$ of the system $W(\mathbb{R} \oplus \mathbb{R}, 0) \otimes W\left(S^{\prime}, \sigma_{2}\right)$ one can characterise two Bose fields $F^{a}$ (where $a$ refers to the abelian character) and $F^{-\frac{1}{2 d}}$ such that

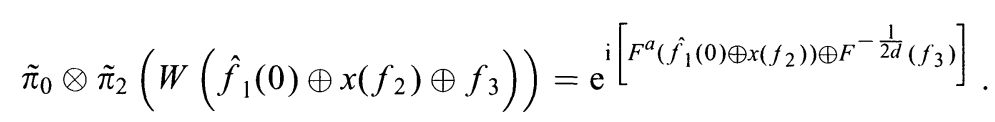


As usual, exactly these Bose field operators $F^{a}\left(\hat{f}_{1}(0) \oplus x\left(f_{2}\right)\right)$ and $F^{-\frac{1}{2 d}}\left(f_{3}\right)$ are defined to be the macroscopic fluctuation observables for this system.

This finishes the discussion of the zero mode fluctuations of the field operators for all the points $(T, \bar{\rho})$ of the phase diagram of a $d$-dimensional free Bose gas.

\section{References}

1. Abramowitz, M., Stegun, I.A.: Handbook of mathematical functions. New York: Dover Publications 1972

2. Araki, H., Woods, E.J.: J. Math. Phys. 4, 637-662 (1963)

3. Buffet, E., Pulè, J.V.: J. Math. Phys. 24, 1608-1616 (1983)

4. Bratteli, O., Robinson, D.: Operator Algebras and Quantum Statistical Mechanics. Vol. I \& II Berlin, Heidelberg, New York: Springer 1979, 1981

5. Broidioi, M.: J. Math. Phys. 35, 2357-2370 (1994)

6. Broidioi, M.: Abnormal Quantum Fluctuations. Leuven: Ph. D. thesis

7. Broidioi, M., Momont, B., Verbeure, A.: Leuven Preprint KUL-TF-95/2

8. Cannon, J.T.: Commun. Math. Phys. 29, 89-104 (1973)

9. Goderis, D., Verbeure, A., Vets, P.: Prob. Th. Rel. Fields 82, 527-544 (1989)

10. Goderis, D., Vets, P.: Commun. Math. Phys. 122, 249-265 (1989)

11. Goderis, D., Verbeure, A., Vets, P.: Commun. Math. Phys. 128, 533-549 (1990)

12. Lanford, O.E., Ruelle, D.: Commun. Math. Phys. 13, 194 (1969)

13. Lewis, J.T., Pulè, J.V.: Commun. Math. Phys. 36, 1-18 (1974)

14. van den Berg, M.: J. Math. Phys. 22, 2452-2455 (1981)

15. Verbeure, A., Zagrebnov, V.A.: J. Stat. Phys. 69, 329-359 (1992)

Communicated by H. Araki 